\title{
PAPER \\ Performance Evaluation of Multiuser MIMO E-SDM Systems in Time-Varying Fading Environments
}

\author{
Huu Phu BUI $^{\dagger \mathrm{a})}$, Yasutaka OGAWA ${ }^{\dagger \mathrm{b})}$, Toshihiko NISHIMURA ${ }^{\dagger \mathrm{c})}$, and Takeo OHGANE $^{\dagger \mathrm{d})}$, Members $^{\text {Ong }}$
}

\begin{abstract}
SUMMARY In this paper, the performance of multiuser MIMO ESDM systems in downlink transmission is evaluated in both uncorrelated and correlated time-varying fading environments. In the ideal case, using the block diagonalization scheme, inter-user interference can be completely eliminated at each user; and using the E-SDM technique for each user, optimal resource allocation can be achieved, and spatially orthogonal substreams can be obtained. Therefore, a combination of the block diagonalization scheme and the E-SDM technique applied to multiuser MIMO systems gives very good results. In realistic environments, however, due to the dynamic nature of the channel and processing delay at both the transmitter and the receiver, the channel change during the delay may cause inter-user interference even if the BD scheme is used. In addition, the change may also result in large inter-substream interference and prevent optimal resource allocation from being achieved. As a result, system performance may be degraded seriously. To overcome the problem, we propose a method of channel extrapolation to compensate for the channel change. Applying our proposed method, simulation results show that much better system performance can be obtained than the conventional case. Moreover, it also shows that the system performance in the correlated fading environments is much dependent on the antenna configuration and the angle spread from the base station to scatterers.

key words: multiuser MIMO systems, eigenbeam-space division multiplexing E-SDM, channel extrapolation, time-varying fading environments
\end{abstract}

\section{Introduction}

The use of multiple antennas at both ends of a communication link, commonly referred to as a multiple-input multipleoutput (MIMO) system, has been widely studied and is considered as a prospective technology to provide high data rate transmission and good performance for the dramatically growing wireless communications demands nowadays. For applications such as wireless LANs and cellular telephony, MIMO systems will likely be deployed in environments where a single base station (BS) must communicate with many users simultaneously [1]-[7]. In the downlink transmission scenario, inter-user interference (IUI) occurs at each user due to simultaneous transmission of users' signals from the BS. A very good solution to completely eliminate the IUI to increase system performance is to use a block diagonization (BD) scheme [4]-[6]. This scheme can decompose the multiuser MIMO channel into multiple parallel and

\section{Manuscript received July 25, 2008.}

Manuscript revised January 13, 2009.

${ }^{\dagger}$ The authors are with the Graduate School of Information Science and Technology, Hokkaido University, Sapporo-shi, 0600814 Japan.

a)E-mail: bhphu@m-icl.ist.hokudai.ac.jp

b) E-mail: ogawa@ist.hokudai.ac.jp

c) E-mail: nishim@ist.hokudai.ac.jp

d)E-mail: ohgane@ist.hokudai.ac.jp

DOI: 10.1587/transcom.E92.B.2374 independent single-user MIMO channels by forcing the interference on a user from the remaining users to be zero.

In a single-user MIMO case, it is well known that the MIMO system applying an eigen-beam space division multiplexing (E-SDM) technique, also called Singular Value Decomposition (SVD) based MIMO system or MIMO eigenmode transmission system, is optimum because the optimal resource allocation can be achieved, and spatially orthogonal substreams can be obtained at a receiver [8]-[13]. Therefore, a natural combination of the BD scheme and the E-SDM technique applied to multiuser MIMO systems gives very good results in terms of system performance.

In realistic environments, however, due to the dynamic nature of the channel and the processing delay at both the transmitter and the receiver, a channel change during the delay may cause IUI even if the BD scheme is used. In addition, the change may also result in large inter-substream interference (ISI) and prevent optimal resource allocation from being achieved. As a result, system performance may be seriously degraded.

Taking the problem into account, in this paper, we propose a method of channel extrapolation to compensate for the channel change. In our proposed method, just only two previous channel responses are needed and the maximum Doppler frequency $\left(f_{\mathrm{D}}\right)$ does not need to be known.

Most of the previous studies have investigated and evaluated multiuser MIMO systems in independent and identically distributed (i.i.d.) fading environments, in which none of the MIMO channels are correlated. However, in realistic environments, channel correlations exist and affect the system performance. Unfortunately, to the best of our knowledge, only a few papers have considered the performance of multiuser MIMO systems in such correlated fading environments [2], [3].

In this paper, we evaluated the performance of multiuser MIMO E-SDM systems using the conventional and our proposed methods in both uncorrelated and correlated time-varying Rayleigh fading environments. Hereinafter, the following notations will be used. Boldface uppercase (lowercase) letters denote matrices (column vectors); $\boldsymbol{X}($ or $\boldsymbol{x}) \in \mathbb{C}^{M \times N}$ denotes an $M \times N$ matrix $\boldsymbol{X}$ (or vector $\boldsymbol{x}$ if $N=1$ ) with $M \times N$ complex value elements.

Our paper is organized as follows. In Sect. 2, we describe the multiuser MIMO E-SDM systems. Next, we propose a method of channel prediction in Sect. 3. We compare and evaluate the performance of multiuser MIMO E-SDM 
systems in both uncorrelated and correlated time-varying fading environments in Sect. 4. Conclusions of the paper are given in Sect. 5. Finally, channel correlations in correlated environments are considered in the appendix.

\section{Multiuser MIMO E-SDM Systems}

A block diagram of a multiuser MIMO E-SDM system is shown in Fig. 1, where $N_{\mathrm{T}}$ antennas are located at the base station (BS) and $N_{\mathrm{R} k}$ antennas are located at the $k$-th user, and there are $K$ active users in the system. At the BS, the input data for each user are dynamically divided into substreams. Let $\boldsymbol{s}_{k}(t) \in \mathbb{C}^{L_{k} \times 1}$ represent the transmit data symbol vector for the $k$-th user, where $L_{k}$ is the number of parallel data symbols transmitted simultaneously for the $k$-th user $(k=1, \cdots, K)$ and $L_{k} \leq \min \left(N_{\mathrm{T}}, N_{\mathrm{R} k}\right)$. This data symbol vector is multiplied by a transmit (TX) weight matrix $\boldsymbol{W}_{\mathrm{TX}, k} \in \mathbb{C}^{N_{T} \times L_{k}}$, and then each of the $N_{\mathrm{T}}$ output data is transmitted by each of the $N_{\mathrm{T}} \mathrm{TX}$ antennas.

We assume that the channel is so narrow that no frequency selective fading occurs. This assumption is made to evaluate the effect of time-varying channels to the performance of multiuser MIMO systems apart from the effect of frequency-selective fading. For wide-band transmission, the orthogonal frequency division multiplexing (OFDM) or the orthogonal frequency division multiplexing access (OFDMA) can be employed. For each subcarrier where we can assume a narrow-band channel, the results obtained in this paper are valid for non-coded OFDM and non-coded OFDMA.

At the $k$-th user, the received signals $\boldsymbol{r}_{k}(t) \in \mathbb{C}^{N_{\mathrm{R} k} \times 1}$ can be given by

$$
\begin{aligned}
\boldsymbol{r}_{k}(t)= & \boldsymbol{H}_{k} \sum_{i=1}^{K} \boldsymbol{W}_{\mathrm{TX}, i} \boldsymbol{s}_{i}(t)+\boldsymbol{n}_{k}(t), \\
= & \boldsymbol{H}_{k} \boldsymbol{W}_{\mathrm{TX}, k} \boldsymbol{s}_{k}(t) \\
& +\boldsymbol{H}_{k} \sum_{i=1, i \neq k}^{K} \boldsymbol{W}_{\mathrm{TX}, i} \boldsymbol{s}_{i}(t)+\boldsymbol{n}_{k}(t),
\end{aligned}
$$

where $\boldsymbol{H}_{k} \in \mathbb{C}^{N_{\mathrm{R} k} \times N_{\mathrm{T}}}$ denotes the MIMO channel matrix of the $k$-th user, $\boldsymbol{n}_{k}(t) \in \mathbb{C}^{N_{\mathrm{Rk}} \times 1}$ denotes the noise vector, whose elements are i.i.d. zero mean complex Gaussian random variables with variance $\sigma^{2}$. The first term in (2) is the desired signal, meanwhile the second term is the interference

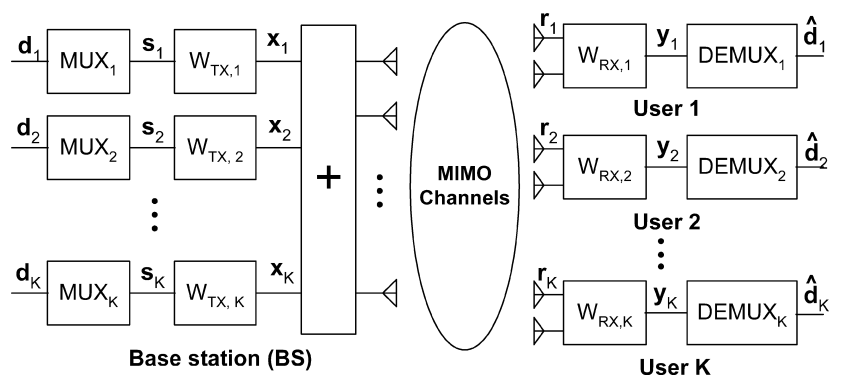

Fig. 1 Model of a multiuser MIMO E-SDM system. from the other $K-1$ users to the $k$-th user. The received signals are demultiplexed by a receive (RX) weight matrix $\boldsymbol{W}_{\mathrm{RX}, k} \in \mathbb{C}^{L_{k} \times N_{\mathrm{Rk}}}$ as follows:

$$
\begin{aligned}
\boldsymbol{y}_{k}(t)= & \boldsymbol{W}_{\mathrm{RX}, k} \boldsymbol{r}_{k}(t) \\
= & \boldsymbol{W}_{\mathrm{RX}, k} \boldsymbol{H}_{k} \boldsymbol{W}_{\mathrm{TX}, k} \boldsymbol{s}_{k}(t) \\
& +\boldsymbol{W}_{\mathrm{RX}, k} \boldsymbol{H}_{k} \sum_{i=1, i \neq k}^{K} \boldsymbol{W}_{\mathrm{TX}, i} \boldsymbol{s}_{i}(t) \\
& +\boldsymbol{W}_{\mathrm{RX}, k} \boldsymbol{n}_{k}(t),
\end{aligned}
$$

where $\boldsymbol{y}_{k}(t) \in \mathbb{C}^{L_{k} \times 1}$ denotes demultiplexed signals.

\subsection{Block Diagonalization Scheme}

To completely eliminate IUI in (2), the TX weight matrices must be selected as follows:

$$
\boldsymbol{H}_{i} \boldsymbol{W}_{\mathrm{TX}, j}=0 \text { for all } i, j: 1 \leq i \neq j \leq K
$$

A BD scheme is described in [4]-[6]. For the scheme, the following condition must be satisfied in order to find the nonzero TX weight matrices:

$$
N_{\mathrm{T}}>\max \left(\sum_{i=1, i \neq k}^{K} N_{\mathrm{R} i} k=1, \cdots, K\right)
$$

Define a matrix as

$$
\hat{\boldsymbol{H}}_{k}=\left[\boldsymbol{H}_{1}^{T} \cdots \boldsymbol{H}_{k-1}^{T} \boldsymbol{H}_{k+1}^{T} \cdots \boldsymbol{H}_{K}^{T}\right]^{T},
$$

where $(\cdot)^{T}$ denotes the matrix transpose. Let $\hat{N}_{k}$ denote the rank of $\hat{\boldsymbol{H}}_{k}\left(\hat{N}_{k} \leq \sum_{i=1, i \neq k}^{K} N_{\mathrm{R} i}\right)$. The SVD of matrix $\hat{\boldsymbol{H}}_{k}$ is given by

$$
\hat{\boldsymbol{H}}_{k}=\hat{\boldsymbol{U}}_{\boldsymbol{k}} \Sigma\left[\hat{\boldsymbol{V}}_{k} \hat{\boldsymbol{V}}_{k}^{0}\right]^{H}
$$

where $(\cdot)^{H}$ denotes the Hermitian matrix transpose, and $\hat{\boldsymbol{V}}_{k}^{0} \in$ $\mathbb{C}^{N_{\mathrm{T}} \times\left(N_{\mathrm{T}}-\hat{N}_{k}\right)}$ contains the last $\left(N_{\mathrm{T}}-\hat{N}_{k}\right)$ right singular vectors of $\hat{\boldsymbol{H}}_{k}$. The columns in $\hat{\boldsymbol{V}}_{k}^{0}$ form a basis set in the null space of $\hat{\boldsymbol{H}}_{k}$, and hence $\boldsymbol{W}_{\mathrm{TX}, k}$ can be any rotated version of $\hat{\boldsymbol{V}}_{k}^{0}$ as

$$
\boldsymbol{W}_{\mathrm{TX}, k}=\hat{\boldsymbol{V}}_{k}^{0} \boldsymbol{T}_{k},
$$

where $\boldsymbol{T}_{k} \in \mathbb{C}^{\hat{N}_{k} \times L_{k}}$ denotes a nonzero arbitrary matrix.

By substituting (4) and (8) into (2), we can obtain

$$
\boldsymbol{r}_{k}(t)=\boldsymbol{H}_{k} \hat{\boldsymbol{V}}_{k}^{0} \boldsymbol{T}_{k} \boldsymbol{s}_{k}(t)+\boldsymbol{n}_{k}(t) .
$$

This means that the multiuser MIMO system has been decoupled to $K$ parallel single-user MIMO systems by using the BD scheme.

\subsection{Eigenbeam-Space Division Multiplexing Technique}

From (9), the equivalent single-user MIMO channel and the equivalent TX weight of the $k$-th user can be represented as 
$\boldsymbol{H}_{\mathrm{eq}, k}=\boldsymbol{H}_{k} \hat{\boldsymbol{V}}_{k}^{0}$ and $\boldsymbol{T}_{k}$, respectively. Applying an E-SDM technique [9], [10] to each equivalent single-user MIMO channel, the equivalent MIMO channel is optimum because the optimal resource allocation can be achieved and spatially orthogonal sub-streams can be obtained at the receiver. In this case, the optimal equivalent TX and RX weights for the $k$-th user can be determined as follows:

$$
\begin{aligned}
& \boldsymbol{T}_{k}=\boldsymbol{V}_{\mathrm{eq}, k} \sqrt{\boldsymbol{P}_{k}}, \\
& \boldsymbol{W}_{\mathrm{RX}, k}=\left(\boldsymbol{H}_{\mathrm{eq}, k} \boldsymbol{V}_{\mathrm{eq}, k}\right)^{H},
\end{aligned}
$$

where $\boldsymbol{P}_{k}=\operatorname{diag}\left(P_{k, 1}, \cdots, P_{k, L_{k}}\right)$ is the TX power matrix for the $k$-th user, and $\boldsymbol{V}_{\text {eq, } k}$ is the right unitary matrix of the SVD of $\boldsymbol{H}_{\mathrm{eq}, k}$ given by

$$
\boldsymbol{H}_{\mathrm{eq}, k}=\boldsymbol{U}_{\mathrm{eq}, k} \sum_{\mathrm{eq}, k} \boldsymbol{V}_{\mathrm{eq}, k}^{H},
$$

where $\sum_{\mathrm{eq}, k}=\operatorname{diag}\left(\lambda_{k, 1}, \cdots, \lambda_{k, L_{k}}\right)$ denotes the diagonal singular value matrix.

Substituting (10) into (8), the TX weight matrix for the $k$-th user is

$$
\boldsymbol{W}_{\mathrm{TX}, k}=\hat{\boldsymbol{V}}_{k}^{0} \boldsymbol{V}_{\mathrm{eq}, k} \sqrt{\boldsymbol{P}_{k}} .
$$

Using (10)-(13), the demultiplexed signals in (3) become as follows:

$$
\boldsymbol{y}_{k}(t)=\sum_{\mathrm{eq}, k}^{2} \sqrt{\boldsymbol{P}_{k}} \boldsymbol{s}_{k}(t)+\boldsymbol{W}_{\mathrm{RX}, k} \boldsymbol{n}_{k}(t) .
$$

This result shows that the E-SDM technique transforms the equivalent single-user MIMO channel into $L_{k}$ orthogonal subchannels. In this paper, we assume that TX power for each user is identical and fixed, and also that the data rate for each user is fixed identically. Under the TX power constraint, by adaptively assigning data resource and transmit power for each stream, the bit-error rate (BER) performance of each user is optimized [9], [10].

\section{Channel Prediction Scheme}

As shown in the above section, in the ideal case, in which the TX weight matrices completely match instantaneous MIMO channels, using the BD scheme, IUI can be completely suppressed. In addition, applying the E-SDM technique to each user, the optimal resource allocation can be achieved and spatially orthogonal subchannels can be obtained. However, in realistic environments, due to the dynamic nature of channels, a channel change during the time interval between the TX weight matrix determination and the actual downlink (DL) data transmission may cause IUI and ISI.

For example, let us consider a 2-user MIMO system with $4 \mathrm{TX}$ antennas at the BS and $2 \mathrm{RX}$ antennas at each user. These users are moving at a constant velocity $v$. When the time interval does not exist (for the ideal case), based on (3) the demultiplexed signals at user 1 can be written as

$$
\begin{aligned}
\boldsymbol{y}_{1}(t)= & \boldsymbol{W}_{\mathrm{RX}, 1}(t) \boldsymbol{H}_{1}(t) \boldsymbol{W}_{\mathrm{TX}, 1}(t) \boldsymbol{s}_{1}(t) \\
& +\boldsymbol{W}_{\mathrm{RX}, 1}(t) \boldsymbol{H}_{1}(t) \boldsymbol{W}_{\mathrm{TX}, 2}(t) \boldsymbol{s}_{2}(t) \\
& +\boldsymbol{W}_{\mathrm{RX}, 1}(t) \boldsymbol{n}_{1}(t) .
\end{aligned}
$$

When the time interval exists (for the realistic case), the demultiplexed signals are given by

$$
\begin{aligned}
\boldsymbol{y}_{1}(t+\tau)= & \boldsymbol{W}_{\mathrm{RX}, 1}(t) \boldsymbol{H}_{1}(t+\tau) \boldsymbol{W}_{\mathrm{TX}, 1}(t) \boldsymbol{s}_{1}(t) \\
& +\boldsymbol{W}_{\mathrm{RX}, 1}(t) \boldsymbol{H}_{1}(t+\tau) \boldsymbol{W}_{\mathrm{TX}, 2}(t) \boldsymbol{s}_{2}(t) \\
& +\boldsymbol{W}_{\mathrm{RX}, 1}(t) \boldsymbol{n}_{1}(t+\tau),
\end{aligned}
$$

where $\tau$ denotes the time interval.

Assuming that the velocity of these users are $5.77 \mathrm{~m} / \mathrm{s}$, which corresponds to $f_{\mathrm{D}}$ of $100 \mathrm{~Hz}$ at the carrier frequency of 5.2 GHz, and the BS transmits two QPSK streams to each user in the downlink channel. At time $t$, MIMO channel matrices of two users are assumed to be as follows:

$$
\begin{aligned}
& \boldsymbol{H}_{1}^{T}(t)=\left[\begin{array}{cc}
0.63-j 2.02 & -1.48+j 0.83 \\
0.99-j 1.11 & -0.17-j 0.56 \\
-0.21+j 0.36 & 0.28+j 0.38 \\
0.05-j 1.14 & -0.58-j 1.38
\end{array}\right] \\
& \boldsymbol{H}_{2}^{T}(t)=\left[\begin{array}{cc}
-0.14-j 0.30 & 0.02+j 0.09 \\
0.98-j 1.90 & 0.09+j 0.73 \\
0.10+j 0.01 & 1.56+j 0.18 \\
0.11+j 0.82 & -0.80+j 0.26
\end{array}\right]
\end{aligned}
$$

Based on Sect. 2, $\boldsymbol{W}_{\mathrm{RX}, 1}(t), \boldsymbol{W}_{\mathrm{TX}, 1}(t)$, and $\boldsymbol{W}_{\mathrm{TX}, 2}(t)$ can be determined. Then, replacing them into (15), the demultiplexed signals at user 1 become:

$$
\begin{aligned}
\boldsymbol{y}_{1}(t)= & {\left[\begin{array}{cc}
3.51 & 0 \\
0 & 1.78
\end{array}\right] \boldsymbol{s}_{1}(t)+\left[\begin{array}{ll}
0 & 0 \\
0 & 0
\end{array}\right] \boldsymbol{s}_{2}(t) } \\
& +\boldsymbol{W}_{\mathrm{RX}, 1}(t) \boldsymbol{n}_{1}(t)
\end{aligned}
$$

This is the result for the ideal case. The above zero matrix means that IUI is completely eliminated, and the diagonal matrix indicates that there is no ISI in user 1.

For the realistic case, assuming that the time interval $\tau=0.25 \mathrm{~ms}$, the MIMO channel matrices of two users at time $t+0.25$ are given by

$$
\begin{aligned}
\boldsymbol{H}_{1}^{T}(t+0.25) & =\left[\begin{array}{cc}
0.61-j 1.91 & -1.50+j 0.90 \\
1.05-j 1.10 & -0.29-j 0.53 \\
-0.30-j 0.19 & 0.32+j 0.54 \\
0.01-j 1.08 & -0.63-j 1.37
\end{array}\right] \\
\boldsymbol{H}_{2}^{T}(t+0.25) & =\left[\begin{array}{cc}
-0.07-j 0.42 & -0.09+j 0.03 \\
0.99-j 2.02 & 0.03+j 0.75 \\
0.06+j 0.12 & 1.579+j 0.151 \\
-0.03+j 0.76 & -0.81+j 0.15
\end{array}\right]
\end{aligned}
$$

They were obtained using Eq. (24) described later in Sect. 4. Based on Eq. (16), the demultiplexed signals at user 1 become:

$$
\begin{aligned}
\boldsymbol{y}_{1}(t+0.25) & =\left[\begin{array}{cc}
3.39-j 0.12 & -0.15-j 0.02 \\
0.04-j 0.06 & 1.77+j 0.02
\end{array}\right] \boldsymbol{s}_{1}(t) \\
& +\left[\begin{array}{cc}
0.18-j 0.04 & -0.41+j 0.28 \\
-0.05-j 0.04 & 0.08+j 0.03
\end{array}\right] \boldsymbol{s}_{2}(t) \\
& +\boldsymbol{W}_{\mathrm{RX}, 1}(t) \boldsymbol{n}_{1}(t+0.25)
\end{aligned}
$$




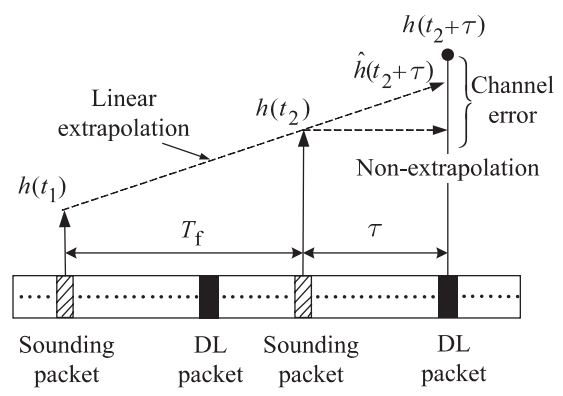

Fig. 2 Linear channel extrapolation scheme.

Here, unlike the ideal case, the second matrix is not a zero one. This implies that IUI from user 2 occurs. In addition, the first matrix is not a diagonal one. This indicates that two subchannels are not orthogonal each other, thus ISI exists. Consequently, the IUI and ISI degrade the system performance.

Taking the problem into account, we propose a method of channel extrapolation to reduce the effect of channel change. In our proposed method, just only two previous channel responses are needed and $f_{\mathrm{D}}$ does not need to be known.

In this study, a time division duplexing (TDD) system ${ }^{\dagger}$ such as HIPERLAN/2 [14], [15] is considered. It is assumed that sounding packets are sent from each user periodically in the TDD uplink channels, and the channel state information (CSI) of each user is then estimated at the base station. ACK packet can be used when it is transmitted. This study also assumes that there is error-free transmission, i.e., sounding packets are always obtained perfectly.

In our proposed method, we first estimate the channel using the last two successive uplink sounding packets. The channel is linearly extrapolated to the actual DL transmission time as shown in Fig. 2, and the predicted value is given by

$$
\hat{h}_{i j, k}\left(t_{2}+\tau\right)=h_{i j, k}\left(t_{2}\right)+\frac{\tau}{T_{\mathrm{f}}}\left(h_{i j, k}\left(t_{2}\right)-h_{i j, k}\left(t_{1}\right)\right),
$$

where $T_{\mathrm{f}}$ is the frame duration of the TDD system, $\tau$ is the time interval between the TX weight matrix determination and the actual DL packet transmission, $h_{i j, k}\left(t_{1}\right)$ and $h_{i j, k}\left(t_{2}\right)$ are the observed channel values from the $j$-th TX antenna of the BS to the $i$-th RX antenna of the $k$-th user at times $t_{1}$ and $t_{2}$, respectively. Also, $\hat{h}_{i j, k}\left(t_{2}+\tau\right)$ is the predicted channel value for the actual DL transmission time $t_{2}+\tau$. Note that the simplest method of obtaining the channel for the DL packet is not to extrapolate the channel but to use $h_{i j, k}\left(t_{2}\right)$. We consider that this is the conventional method without the extrapolation.

\section{Performance Analyses}

\subsection{Simulation Parameters}

Simulation parameters are shown in Table 1 . Here, we assumed that $N_{\mathrm{R}, 1}=\cdots=N_{\mathrm{R}, K}=N_{\mathrm{R}}$ and $N_{\mathrm{T}}=N_{\mathrm{R}} \times K$. Data
Table 1 Simulation parameters for multiuser MIMO E-SDM system.

\begin{tabular}{|c|c|}
\hline No of users $(K)$ & 2,3 \\
\hline $\begin{array}{l}\text { No of TX } \times \mathrm{RX} \text { antennas } \\
\left(N_{\mathrm{T}} \times N_{\mathrm{R}}\right)\end{array}$ & $(4 \times 2)(6 \times 2)(6 \times 3)(9 \times 3)$ \\
\hline $\begin{array}{l}\text { Array configuration } \\
\text { in the correlated case }\end{array}$ & $\begin{array}{c}\text { Uniform linear array with } \\
\text { antenna spacing of } 0.5 \lambda \& 1.0 \lambda \\
\text { and } \mathrm{TX}-x / \mathrm{RX}-x \& \mathrm{TX}-y / \mathrm{RX}-y\end{array}$ \\
\hline Data rates & $\begin{array}{l}4 \mathrm{bps} / \mathrm{Hz}\left(N_{\mathrm{R}}=2\right) \\
6 \mathrm{bps} / \mathrm{Hz}\left(N_{\mathrm{R}}=3\right)\end{array}$ \\
\hline Modulation schemes & 64QAM, 16QAM, QPSK \\
\hline Resource control & $\begin{array}{c}\text { Minimum BER criterion } \\
\text { based on Chernoff upper-bound }\end{array}$ \\
\hline Data burst length & 128 symbols \\
\hline No of burst trial & 100,000 \\
\hline Frame duration $\left(T_{\mathrm{f}}\right)$ & $2.0 \mathrm{~ms}$ \\
\hline Delay from sounding packet & $0.25 \mathrm{~ms}$ \\
\hline Max. Doppler freq. $\left(f_{\mathrm{D}}\right)$ & $20 \& 100 \mathrm{~Hz}$ \\
\hline Channels & Rayleigh fading \\
\hline Angle spread from BS $(\Delta \theta)$ & $\pi / 10 \& \pi / 4$ \\
\hline CSI & Obtained perfectly \\
\hline Thermal noise & Additive White Gaussian noise \\
\hline RX signal processing & MRC \& MMSE \\
\hline
\end{tabular}

rates were fixed constantly at 4 and $6 \mathrm{bps} / \mathrm{Hz}$ (bits per symbol duration) for the case of $N_{\mathrm{R}}=2$ and 3 , respectively. The number of substreams was dependent on the resource adaptation, specifically the modulation scheme and the transmit power. When $N_{\mathrm{R}}=2$, we had two cases of the resource selection, namely: 16QAM $\times 1(1$ stream $)$ and QPSK $\times 2(2$ streams). Meanwhile, when $N_{\mathrm{R}}=3$, three cases with possible resource selection were $64 \mathrm{QAM} \times 1$ (1 stream), 16QAM $\times 1+$ QPSK $\times 1$ ( 2 streams), and QPSK $\times 3$ (3 streams). The reason why we need resource selection is because we should send more bits over a substream with higher SNR and fewer bits over a substream with lower SNR to obtain better BER under the fixed data rate requirement. Thus, we need to determine the modulation scheme for each substream considering SNR that was stated in Sect. 2. Also, we need to allocate the transmit power to each substream properly. The number of substreams, modulation schemes, and power allocation for each user were adaptively determined in such a way that the Chernoff upper bound of BER of each user had the lowest value [9], [10]. The total transmit power per user was assumed to be equal. This study also assumed that the frame duration of the TDD system was $2.0 \mathrm{~ms}$, as in the HIPERLAN/2 standardization [14], [15], and the delay of actual DL data transmission was $0.25 \mathrm{~ms}$.

HIPERLAN/2 [14], [15] was specified by the European Telecommunications Standards Institute - Broadband Radio Access Networks project. Some parameters and concepts of HIPERLAN/2 are assumed in this paper. The system operates at $5 \mathrm{GHz}$ frequency band and can provide data rates of up to $54 \mathrm{Mbps}$ for short-range (up to $150 \mathrm{~m}$ ) communi-

Although a TDD system is considered in the paper, the obtained results are equivalently applied to a frequency division duplex scheme in which CSI is estimated at each user based on pilot signals or training symbols and then fed back to the base station. 
cations in indoor and outdoor environments. The air interface of the HIPERLAN/2 standard is based on TDD and dynamic time-division multiple access (TDMA). The basic frame structure on the air interface has a fixed duration of $2 \mathrm{~ms}$, and the data units to be transmitted via the physical layer are bursts of variable length. The system may be used in some different scenarios such as:

- Infrastructure replacement scenario, i.e., when HIPERLAN/2 could be used instead of cabling.

- Cordless access scenario, in which users need to use HIPERLAN/2 in different locations at different times
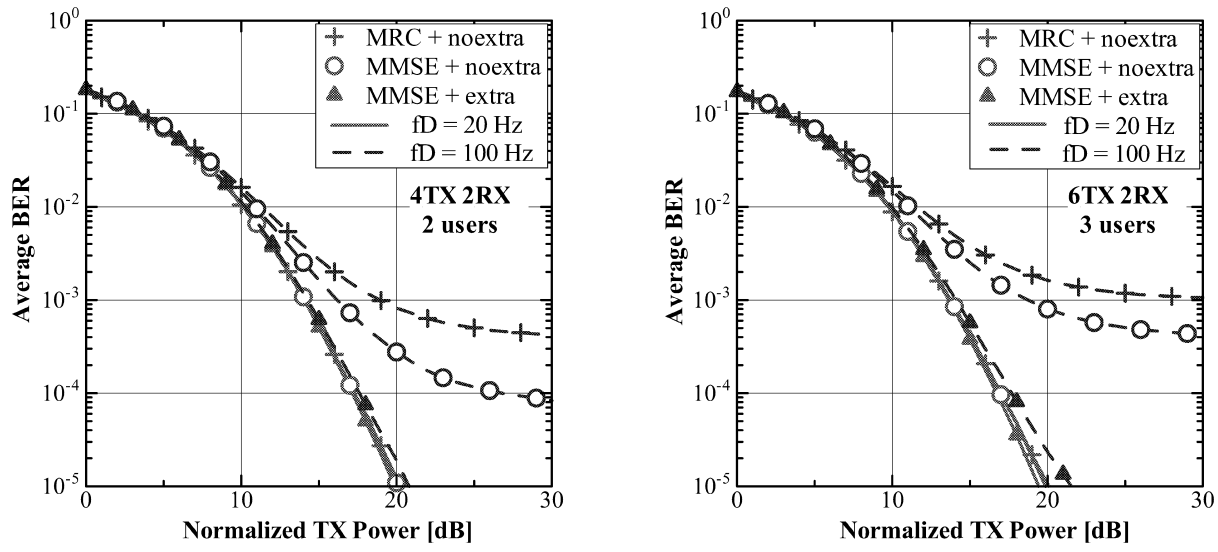

(a) $N_{\mathrm{R}}=2$
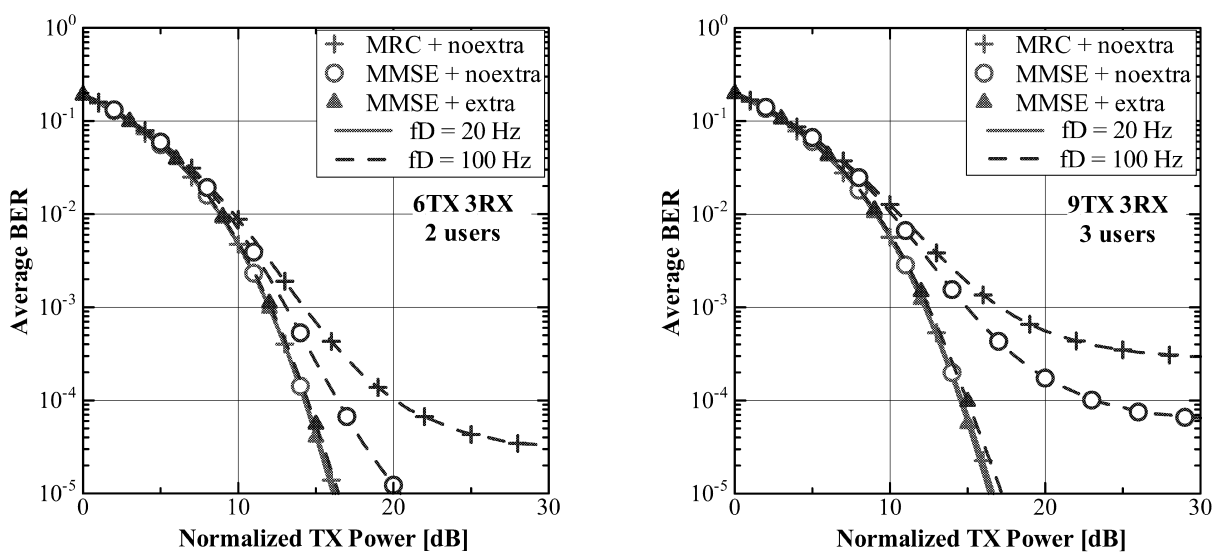

(b) $N_{\mathrm{R}}=3$

Fig. 3 Average BER performance versus normalized TX power in uncorrelated environments when $\left(N_{\mathrm{T}} \times N_{\mathrm{R}} \times K\right)=(4 \times 2 \times 2),(6 \times 2 \times 3),(6 \times 3 \times 2)$, and $(9 \times 3 \times 3)$

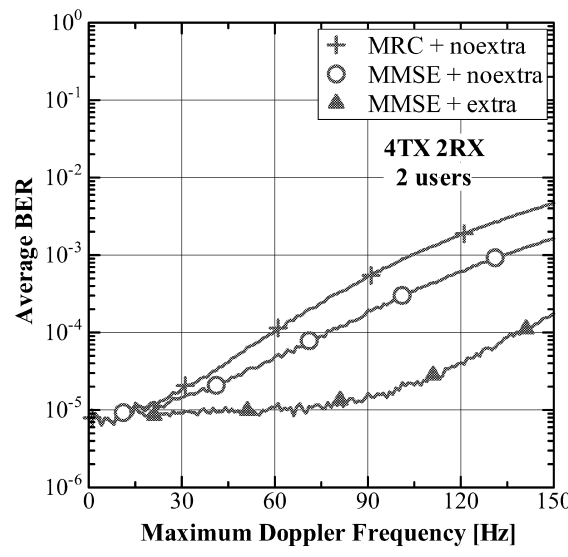

(a) $K=2$

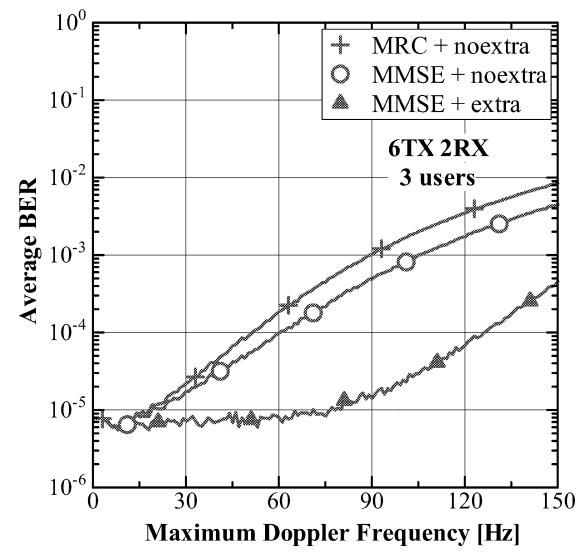

(b) $K=3$

Fig. 4 Average BER performance versus the maximum Doppler frequency at the normalized TX power of $20 \mathrm{~dB}$ in uncorrelated environments when $\left(N_{\mathrm{T}} \times N_{\mathrm{R}} \times K\right)=(4 \times 2 \times 2)$ and $(6 \times 2 \times 3)$. 
possibly maintaining connectivity while in transit.

- Wireless access to infrastructure scenario.

- Specialized portable applications scenario, i.e., user has a personal digital assistant (PDA) type device mainly for specialized applications, e.g. maintenance or surveillance.

- Domestic premises scenario, i.e., HIPERLAN/2 is used in the home environment.

- Wireless manufacturing automation scenario, i.e., HIPERLAN/2 is used in a factory or a large assembly/building facility.

- Internet work communication scenario.

Terminals used in HIPERLAN/2 could be personal computers, portable computers, workstations, PDAs, intelligent transportation elements, autonomous/automatic vehicles, surveillance systems, etc. Depending on scenarios, the mobility of the terminals may be fixed, walking speed, or slow vehicles limited within $10 \mathrm{~m} / \mathrm{s}$ (i.e., the maximum Doppler frequency is less than $173 \mathrm{~Hz}$ for the carrier frequency of $5.2 \mathrm{GHz}$ ). Here, two $f_{\mathrm{D}}$ values of 20 and $100 \mathrm{~Hz}$ were considered, which corresponded to two terminal's velocities of 1.15 and $5.77 \mathrm{~m} / \mathrm{s}$, respectively. The mobility can be considered as walking speed or slow vehicles.

In the paper, it is assumed that the channel state information is obtained perfectly at the BS and the users, and that the packet duration is so short that the channel change within the packet is negligible. The channels are assumed to be constant within a data packet and change between data packets according to the following Eq. (24). This means in this study that channels are assumed to be quasi-static. In the simulation, time-varying flat fading channels based on the Jakes model [16] were considered as follows:

$$
h_{i, j, k}(t)=\frac{1}{M} \sum_{m=1}^{M} e^{j 2 \pi f_{\mathrm{D}}\left(\cos \beta_{m}\right) t+\theta_{i, j, k, m}},
$$

where $M$ denotes the number of scatterers uniformly distributed around each user, $\beta_{m}$ denotes the direction of signal arrival from the $m$-th scatterer, $\theta_{i, j, k, m}$ denotes the random initial phase, and $h_{i, j, k}(t)$ denotes the fading channel from the $j$-th TX antenna to the $i$-th RX antenna of the $k$-th user. From (24), fading channels can be easily determined after the time delay from sounding packet $t=\tau$ at the maximum Doppler frequency $f_{\mathrm{D}}$.

\subsection{Performance in Uncorrelated Environments}

In this subsection, we evaluate the performance of multiuser MIMO E-SDM systems in i.i.d. Rayleigh fading environments, in which there are no correlations between any channels.

The average BER performance versus normalized TX power for $f_{\mathrm{D}}=20$ and $100 \mathrm{~Hz}$ is shown in Fig. 3. The average BER is defined as follows:

$$
\text { Average BER }=\frac{\text { total error bits for each user }}{\text { total transmitted bits for each user }}
$$

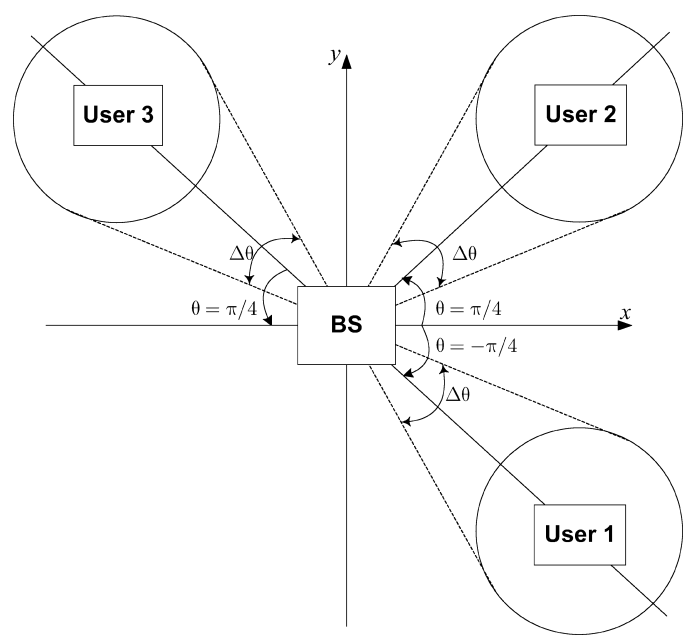

Fig. 5 Positions of BS and users.

$$
=\frac{\text { total error bits for each user }}{100,000 \times 128 \times N_{\mathrm{R}} \times 2}
$$

The normalized TX power is defined as follows: We assume in the paper that antenna arrays at the BS and users consist of omni-directional antenna elements. Normalized TX power is the transmitted power per user that is normalized by the value yielding average $E_{s} / N_{0}$ of $0 \mathrm{~dB}$ at the single omni-directional antenna receiver when a signal is transmitted from the single omni-directional antenna. $E_{s}$ and $N_{0}$ denote the energy received during a symbol interval and thermal noise power spectrum density, respectively. Note that $E_{s} / N_{0}$ is equal to SNR. It is seen that when $N_{\mathrm{R}}$ increases, better performance can be obtained due to higher diversity gain. When $f_{\mathrm{D}}=20 \mathrm{~Hz}$, the performances for the conventional method without the extrapolation (denoted as noextra in Fig. 3) and our proposed extrapolation method are almost the same. This is because the channels changed a little during the time interval $\tau$, therefore introducing small IUI and ISI. Any detection weight, MRC or MMSE, can give good performance. However, when $f_{\mathrm{D}}$ is as high as $100 \mathrm{~Hz}$, the performance for the conventional method is seriously degraded. The reason is that during the time interval $\tau$ the channels had a large change, which resulted in large IUI even if the BD scheme is used, and caused large ISI due to a large loss of subchannel orthogonality for each user. It is also observed that the MMSE weight is superior to the MRC one because MMSE can suppress more interference. On the other hand, in our proposed method, in which the channels are extrapolated to the actual downlink transmission time and MMSE weights are used for detecting received signals, the channel changes during the time interval $\tau$ were compensated, thus IUI and ISI were significantly suppressed and the resource allocation for each user was more adequate. As a result, much better BER performance can be obtained compared with that in the conventional case. It should be noted that although the performance for the ideal case (i.e., $\tau=0$ ) was not shown here, it is almost the same as the performance for the extrapolation case when $f_{\mathrm{D}}=20 \mathrm{~Hz}$. 


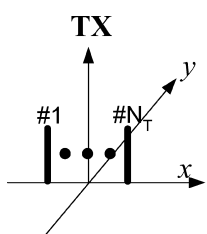

(a) $\mathrm{TX}-x / \mathrm{RX}-x$

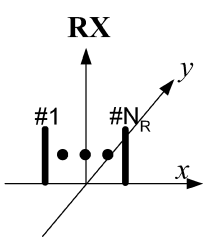

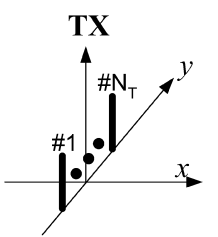

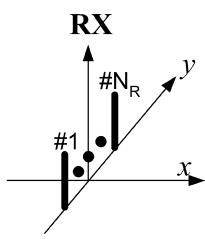

(b) TX-y/RX-y

Fig. 6 Antenna array orientations.

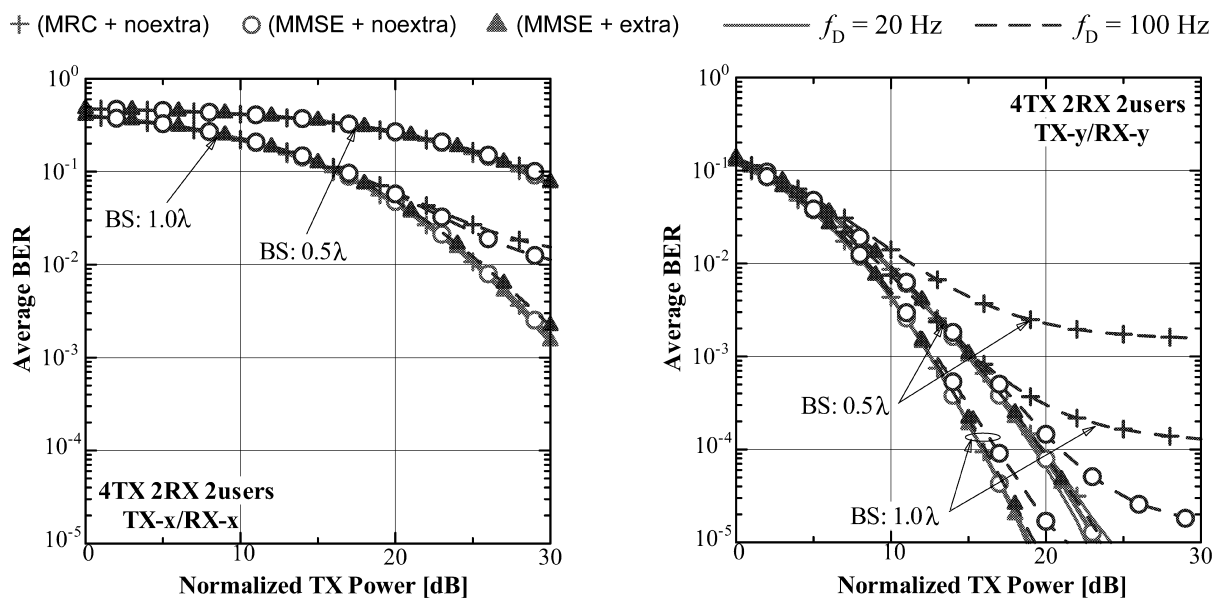

(a) $K=2$
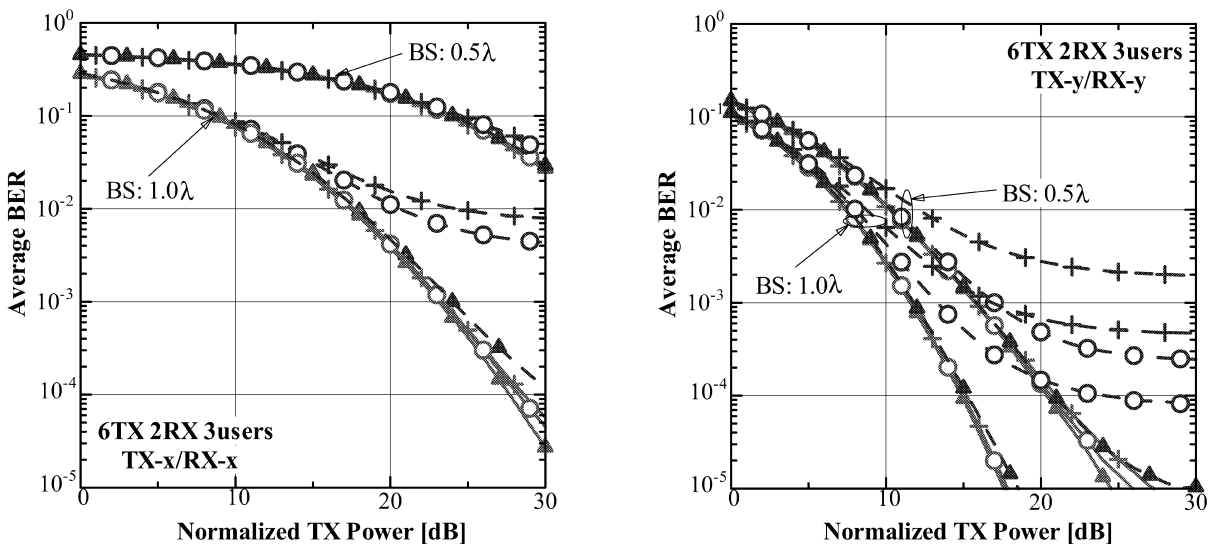

(b) $K=3$

Fig. 7 Average BER performance versus normalized TX power in correlated environments when $\left(N_{\mathrm{T}} \times N_{\mathrm{R}} \times K\right)=(4 \times 2 \times 2),(6 \times 2 \times 3)$, and $\Delta \theta=\pi / 10$.

From Fig. 3, it can be seen that at $f_{\mathrm{D}}=100 \mathrm{~Hz}$, the more the number of users is, the more the performance of the conventional case is degraded due to the larger IUI and ISI; meanwhile our proposed method gives very good results.

Average BER performance versus the maximum Doppler frequency at the normalized TX power of $20 \mathrm{~dB}$ in uncorrelated environments is shown in Fig. 4. It is observed that the performance of the non-extrapolation case is seriously degraded in time-varying fading environments. Meanwhile, using our proposed linear channel extrapolation method, the performance is good in the range of the $f_{\mathrm{D}}$ until about $100 \mathrm{~Hz}$, and begins to be degraded when $f_{\mathrm{D}}$ is above about $100 \mathrm{~Hz}$.

\subsection{Performance in Correlated Environments}

In realistic environments, channel correlations exist between the BS and users. These affect the system performance. Therefore, we investigate the system performance in correlated Rayleigh fading environments. In this subsection the BER performance is examined, while TX and RX channel correlations for two-user case are considered in the appendix.

This study assumed the positions of BS and users shown in Fig. 5. There was a ring of 13 uniformly distributed scatterers surrounding each user, and the angle 

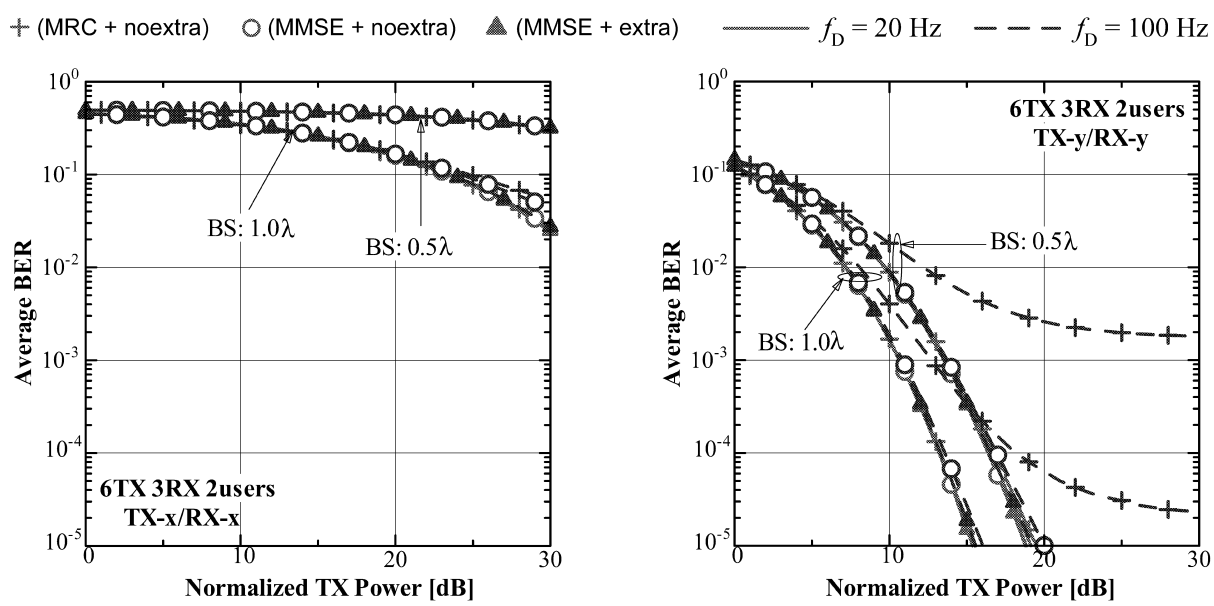

(a) $K=2$
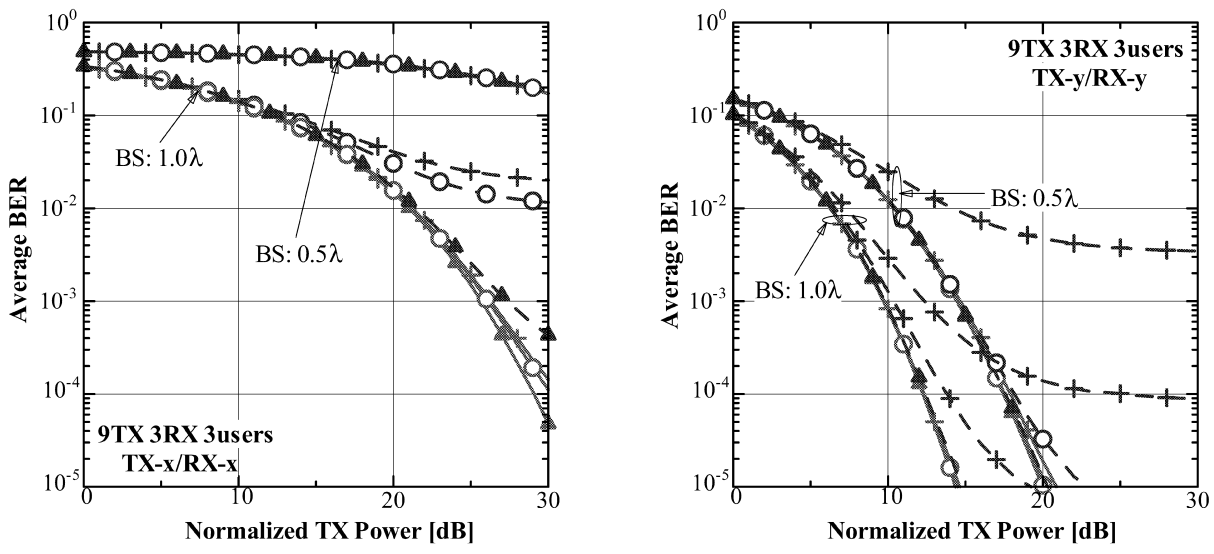

(b) $K=3$

Fig. 8 Average BER performance versus normalized TX power in correlated environments when $\left(N_{\mathrm{T}} \times N_{\mathrm{R}} \times K\right)=(6 \times 3 \times 2),(9 \times 3 \times 3)$, and $\Delta \theta=\pi / 10$.

spread $\Delta \theta$ from the BS to each ring of scatterers was equal to $\pi / 10$ and $\pi / 4$. At the BS and users, uniform linear arrays were considered. Two array orientations as shown in Fig. 6 were examined. One is $\mathrm{TX}-x / \mathrm{RX}-x$, in which the antenna arrays at the BS and users were along the $x$-axis. The other is TX- $y / \mathrm{RX}-y$, in which the arrays at the BS and users were along the $y$-axis. It should be noted that $x$ - and $y$-axes were defined as shown in Fig. 5. In addition, two cases of antenna spacing (AS) were examined such that $1-$ AS between adjacent elements was half of the wavelength $(0.5 \lambda)$ at all the BS and users, and 2- AS was $1.0 \lambda$ at the BS, and $0.5 \lambda$ at all the users.

The average BER performance of user 1 for $f_{\mathrm{D}}=20$ and $100 \mathrm{~Hz}$ in the correlated environments when $\left(N_{\mathrm{T}} \times N_{\mathrm{R}} \times\right.$ $K)=(4 \times 2 \times 2),(6 \times 2 \times 3)$, and $\Delta \theta=\pi / 10$ is shown in Fig. 7. The performance is much dependent on the antenna configuration. In both cases of $K=2$ and 3, better performance can be obtained for the TX- $y / \mathrm{RX}-y$ orientation than for the TX- $x / \mathrm{RX}-x$ orientation. This is due to the lower TX and RX inter-user channel correlations with the TX- $y / \mathrm{RX}-y$ orientation as shown in Tables A. 1 and A. 5 in the appendix. It is interesting that when the AS at the BS is extended from
$0.5 \lambda$ to $1.0 \lambda$, better performance gain can be obtained for the the TX- $x / \mathrm{RX}-x$ orientation than for the TX- $y / \mathrm{RX}-y$ orientation. The reason for this is that both TX and RX interuser channel correlations with the TX- $x / \mathrm{RX}-x$ orientation were considerably reduced with the extention; meanwhile with the TX- $y / \mathrm{RX}-y$ orientation, the TX correlations were decreased, but the RX inter-user ones were not as shown in Tables A. 1 and A.5. As in the uncorrelated environments, performance of the conventional and our proposed methods are almost the same at $f_{\mathrm{D}}=20 \mathrm{~Hz}$; however, in the case of $f_{\mathrm{D}}=100 \mathrm{~Hz}$, our proposed method outperforms the conventional one.

The average BER performance of user 1 for $f_{\mathrm{D}}=20$ and $100 \mathrm{~Hz}$ when $\left(N_{\mathrm{T}} \times N_{\mathrm{R}} \times K\right)=(6 \times 3 \times 2),(9 \times 3 \times 3)$, and $\Delta \theta=\pi / 10$ is shown in Fig. 8. The same tendency can be observed as in the above described case of $\left(N_{\mathrm{T}} \times N_{\mathrm{R}} \times K\right)=$ $(4 \times 2 \times 2),(6 \times 2 \times 3)$. In addition, compared with the results as shown in Fig. 7, worse performance can be seen for the TX- $x / \mathrm{RX}-x$ orientation, but better performance can be obtained for the TX-y/RX- $y$ orientation. This is because the TX and RX channel correlations with the TX- $x / \mathrm{RX}-x$ orientation were very high as shown in Tables A. 3 and A. 7 , 

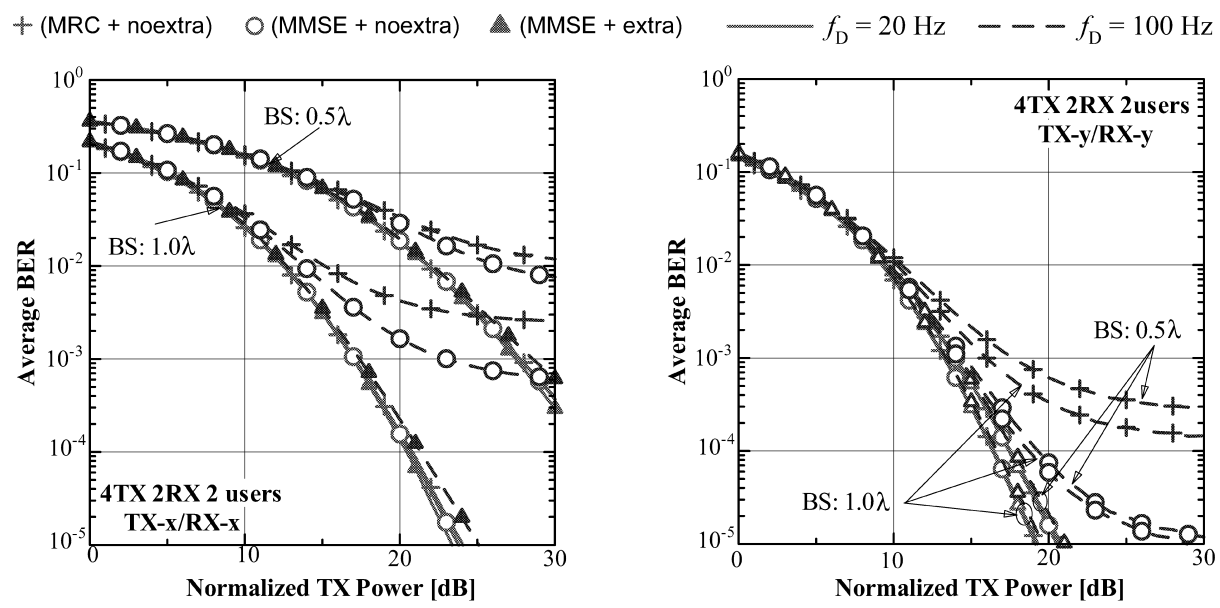

(a) $K=2$
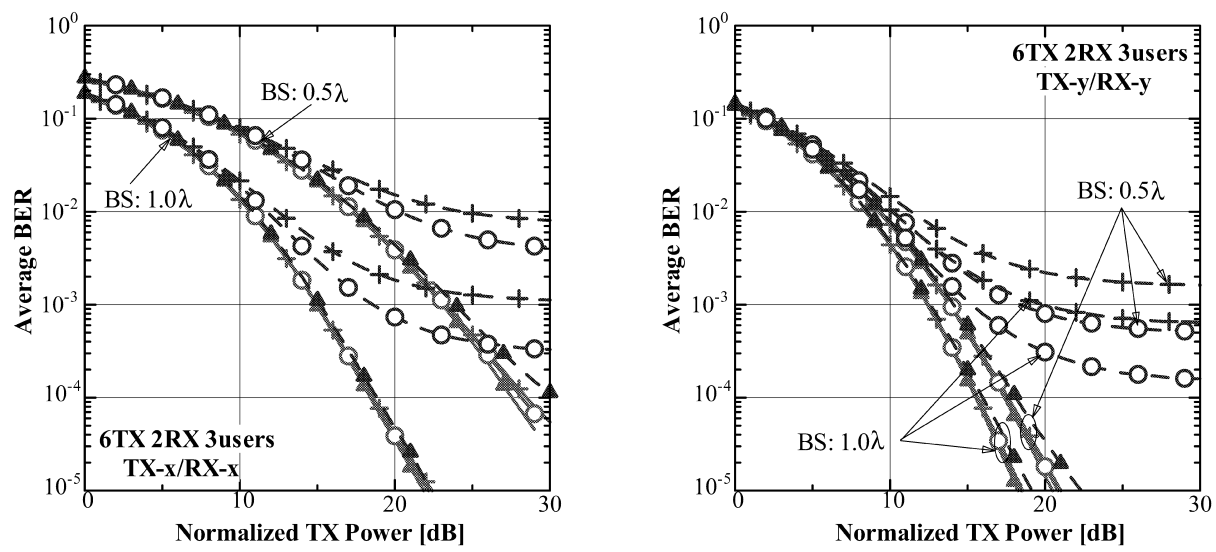

(b) $K=3$

Fig. 9 Average BER performance versus normalized TX power in correlated environments when $\left(N_{\mathrm{T}} \times N_{\mathrm{R}} \times K\right)=(4 \times 2 \times 2),(6 \times 2 \times 3)$, and $\Delta \theta=\pi / 4$.

thus diversity gain seemed to be not effective. On the other hand, due to the low TX and RX channel correlations, the diversity gain is obtained for the TX- $y / \mathrm{RX}-y$ orientation.

The average BER performance of user 1 for $f_{\mathrm{D}}=20$ and $100 \mathrm{~Hz}$ when $\left(N_{\mathrm{T}} \times N_{\mathrm{R}} \times K\right)=(4 \times 2 \times 2),(6 \times 2 \times$ $3),(6 \times 3 \times 2),(9 \times 3 \times 3)$, and $\Delta \theta=\pi / 4$ is shown in Figs. 9 and 10 . Here, it is also observed that the performance is much dependent on the antenna configuration as discussed previously. Furthermore, it is also seen that when the angle spread from the BS to the ring of scatterers increases, better performance can be obtained. This obviously results from the lower TX and RX inter-user channel correlations as shown in Tables A.2, A.4, A. 6, and A. 8 .

Average BER performance of user 1 versus the maximum Doppler frequency at the normalized TX power of $20 \mathrm{~dB}$ when $\left(N_{\mathrm{T}} \times N_{\mathrm{R}} \times K\right)=(4 \times 2 \times 2) \&(6 \times 2 \times$ 3), $\Delta \theta=\pi / 10 \& \pi / 4$, and AS $=0.5 \lambda$ is shown in Fig. 11 . For the TX- $x / \mathrm{RX}-x$ orientation, the performances of the non-extrapolation case and our proposed method are not much different. However, for the TX- $y / \mathrm{RX}-y$ orientation, the difference between our proposed method and the nonextrapolation case is shown very clearly, and our proposed method is considerably superior to the non-extrapolation case. The reason for this is that channel correlations are high for the TX- $x / \mathrm{RX}-x$ orientation, thus the channels change similarly. In this case, although the diversity effect is small, the effect of the channel tracking error is not serious. On the other hand, for the TX-y/RX- $y$ orientation, channel correlations are lower and thus better diversity can be obtained. However, channels change more rapidly during the time interval $\tau$. This causes the performance of the nonextrapolation case to be seriously degraded. On the other hand, by compensating for the channel change, performance of our proposed method is much better. Here, it is observed that our proposed method can be applied in the range of the $f_{\mathrm{D}}$ until about $120 \mathrm{~Hz}$.

In this paper, the time delay $\tau$ is assumed to be $0.25 \mathrm{~ms}$. Simulation results have shown that the proposed linear channel extrapolation method is superior to the conventional case (non-extrapolation case). However, when the delay is longer and/or the maximum Doppler frequency is higher than $100 \mathrm{~Hz}$ for the uncorrelated case or $120 \mathrm{~Hz}$ for the correlated case, inter-user interference and inter-subchannel interference are larger. Applying the proposed linear extrapo- 

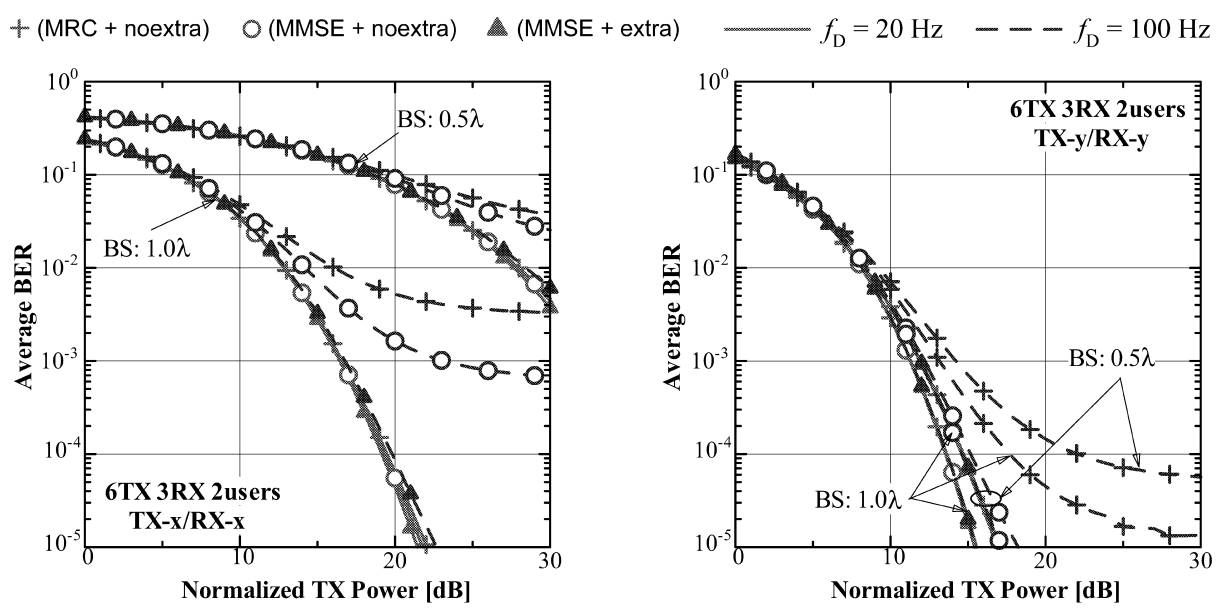

(a) $K=2$
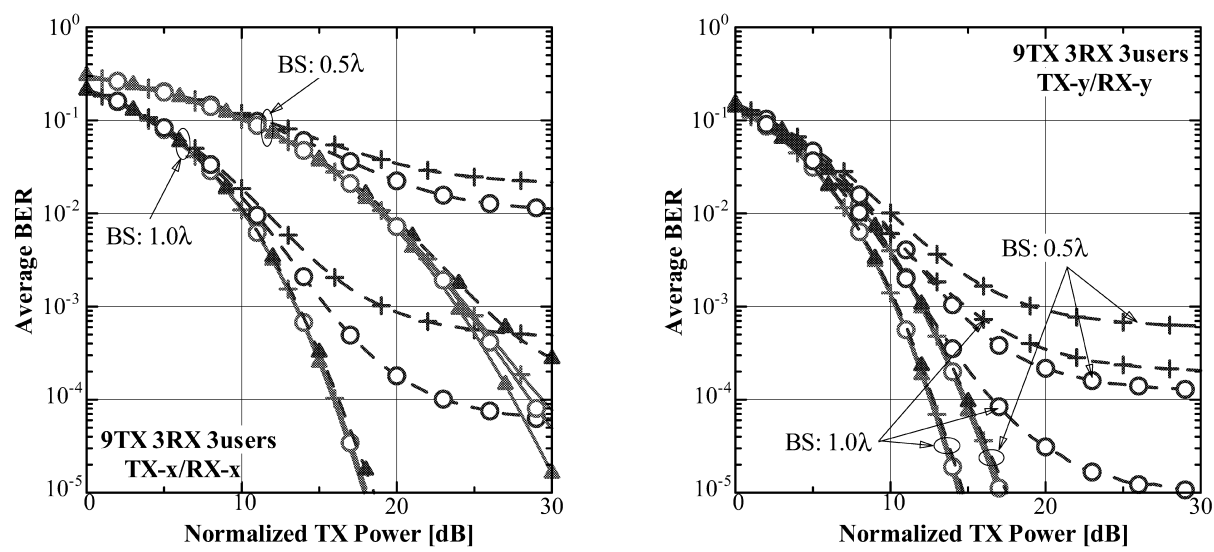

(b) $K=3$

Fig. 10 Average BER performance versus normalized TX power in correlated environments when $\left(N_{\mathrm{T}} \times N_{\mathrm{R}} \times K\right)=(6 \times 3 \times 2),(9 \times 3 \times 3)$, and $\Delta \theta=\pi / 4$.

lation method, the system performance may be degraded. In such a case, instead of using the linear method, the two different extrapolation methods proposed by the authors [11], the second-order extrapolation or the exponential extrapolation, can be employed to improve the system performance.

\section{Conclusion}

In this paper, the performance of multiuser MIMO E-SDM systems was evaluated in both uncorrelated and correlated time-varying fading environments. In the environments, due to a channel change during the time interval between the TX weight matrix determination and the actual DL data transmission, the IUI and ISI were not completely eliminated even if the BD scheme and the E-SDM technique were used. As a result, simulation results based on computer-generated data showed that the system performance can be seriously degraded. To overcome the problem, we have proposed a method of channel extrapolation to compensate for the channel change. Applying our proposed method, the results showed that the performance can be significantly increased. Moreover, it was also shown that the system performance in the correlated fading environments was much dependent on the antenna configuration and the angle spread from the base station to scatterers.

In this paper, the performance of multiuser MIMO systems have just been evaluated in narrow-band flat fading environments. For wide-band transmission, frequencyselective fading channels exist and cause inter-symbol interference. It is well-known that OFDM, OFDMA, and single carrier modulation with frequency domain equalization are common techniques to deal with the interference. As stated in Sect. 2, the results obtained in this paper are valid for each subcarrier in non-coded OFDM or non-coded OFDMA systems. However, the extension of the scheme described in this paper to realistic wide-band systems is future work.

\section{Acknowledgement}

The authors would like to thank the reviewers for reviewing the manuscript and giving us crutial comments. 
$+($ MRC + noextra $) \quad O($ MMSE + noextra $) \quad$ A (MMSE + extra $)$
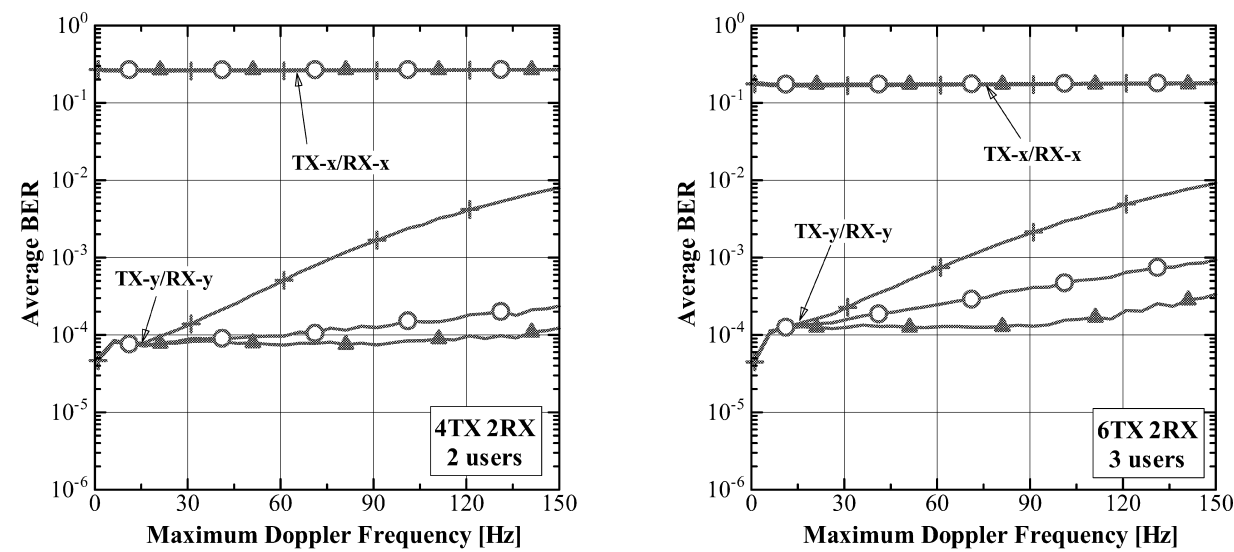

(a) $\Delta \theta=\pi / 10$
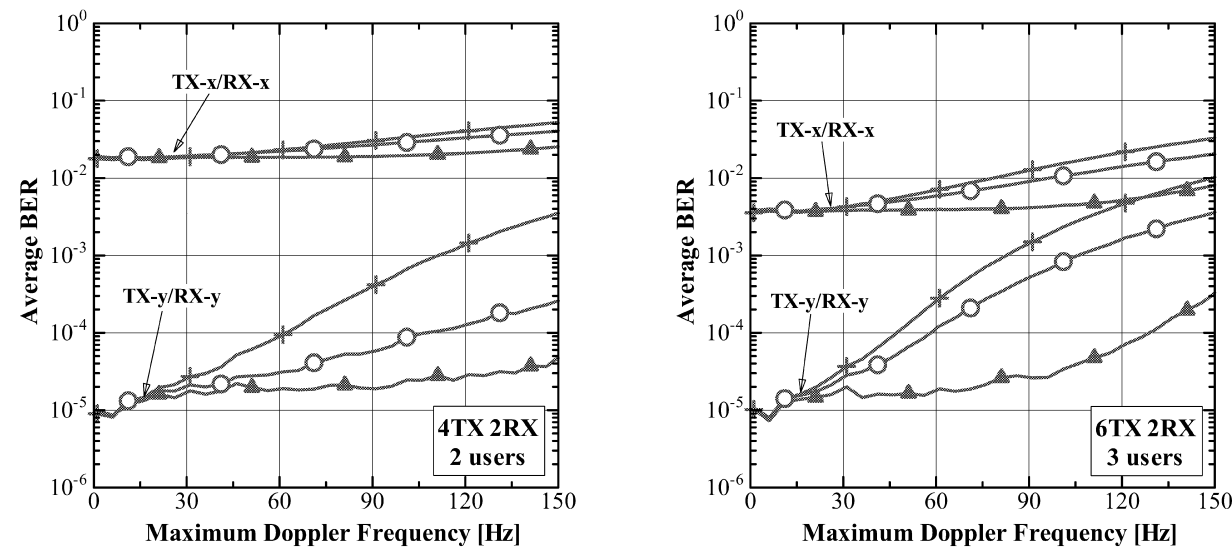

(b) $\Delta \theta=\pi / 4$

Fig. 11 Average BER performance versus the maximum Doppler frequency at the normalized TX power of $20 \mathrm{~dB}$ in correlated environments when $\left(N_{\mathrm{T}} \times N_{\mathrm{R}} \times K\right)=(4 \times 2 \times 2) \&(6 \times 2 \times 3), \Delta \theta=$ $\pi / 10 \& \pi / 4$, and $\mathrm{AS}=0.5 \lambda$

\section{References}

[1] K.K. Wong, R.D. Murch, and K.B. Letaief, "A joint-channel diagonalization for multiuser MIMO antenna systems," IEEE Trans. Wireless Commun., vol.2, no.4, pp.773-786, July 2003.

[2] C. Pietsch, S. Sand, G. Teich, and J. Lindner, "Modeling and performance evaluation of multiuser MIMO systems using real-valued matrices,” IEEE J. Sel. Areas Commun., vol.21, no.5, pp.744-753, June 2003.

[3] J. Chung, C.S. Hwang, K. Kim, and Y.K. Kim, "A random beamforming technique in MIMO systems exploiting multiuser diversity," IEEE J. Sel. Areas Commun., vol.21, no.5, pp.848-855, June 2003.

[4] L.U. Choi and R.D. Murch, "A transmit preprocessing technique for multiuser MIMO systems using a decomposition approach," IEEE Trans. Wireless Commun., vol.3, no.1, pp.20-24, Jan. 2004.

[5] Q.H. Spencer, A.L. Swindlehurst, and M. Haardt, "Zero-forcing methods for downlink spatial multiplexing in multiuser MIMO channels," IEEE Trans. Signal Process., vol.52, no.2, pp.461-471, Feb. 2004.

[6] Q.H. Spencer, C.B. Peel, A.L. Swindlehurst, and M. Haardt, "An introduction to the multi-user MIMO downlink," IEEE Commun. Mag., vol.42, no.10, pp.60-67, Oct. 2004.

[7] M. Sadek, A. Tarighat, and A.H. Sayed, "A leakage-based precoding scheme for downlink multi-user MIMO channels," IEEE Trans.
Wireless Commun., vol.6, no.3, pp.1711-1721, May 2007.

[8] E. Telatar, "Capacity of multi-antenna Gaussian channels," European Transaction on Telecommunications, vol.10, no.6, pp.585-589, Nov./Dec. 1999.

[9] K. Miyashita, T. Nishimura, T. Ohgane, Y. Ogawa, Y. Takatori, and K. Cho, "High data-rate transmission with eigenbeam-space division multiplexing (E-SDM) in a MIMO channel," Proc. IEEE VTC 2002Fall, vol.3, pp.1302-1306, Sept. 2002.

[10] T. Ohgane, T. Nishimura, and Y. Ogawa, "Applications of space division multiplexing and those performance in a MIMO channel," IEICE Trans. Commun., vol.E88-B, no.5, pp.1843-1851, May 2005.

[11] H.P. Bui, Y. Ogawa, T. Ohgane, and T. Nishimura, "Channel extrapolation techniques for E-SDM system in time-varying fading environments," IEICE Trans. Commun., vol.E89-B, no.11, pp.30383092, Nov. 2006.

[12] G. Labrun, J. Gao, and M. Faulkner, "MIMO transmission over a time-varying channel using SVD," IEEE Trans. Wireless Commun., vol.4, no.2, pp.757-764, March 2005.

[13] S.H. Ting, K. Sakaguchi, and K. Araki, "A robust and low complexity adaptive algorithm for MIMO eigenmode transmission system with experimental validation," IEEE Trans. Wireless Commun., vol.5, no.7, pp.1775-1784, July 2006.

[14] TR 101031 V2.2.1 (1999-01) "Broadband Radio Access Networks (BRAN); HIgh PErformance Radio Local Area Network (HIPER- 
LAN) Type 2; Requirements and architectures for wireless broadband access," ETSI Technical Report, Jan. 1999.

[15] J. Khun-Jush, G. Malmgren, P. Schramm, and J. Torsner, "HIPERLAN type 2 for broadband wireless communications," Ericsson Review Mag., vol., no.2, pp.108-119, 2000.

[16] W.C. Jakes, Microwave Mobile Communications, John Wiley \& Sons, 1974

\section{Appendix: Channel Correlations}

In realistic environments, channel correlations exist between the BS and users. These affect the system performance. Therefore, in this appendix, the TX channel correlations between TX antennas at the BS and the RX channel correla- tions between RX antennas in each user and inter-users are considered in the correlated environments as described in Sect. 4.3.

The TX channel correlation $\rho_{\mathrm{TX}, i j}$ between the $i$-th and $j$-th TX antennas at the BS is given by

$$
\rho_{\mathrm{TX}, i j}=\frac{\sum_{k=1}^{K} \sum_{r=1}^{N_{\mathrm{R}}} h_{r i, k}^{*} h_{r j, k}}{\sqrt{\sum_{k=1}^{K} \sum_{r=1}^{N_{\mathrm{R}}}\left|h_{r i, k}\right|^{2}} \sqrt{\sum_{k=1}^{K} \sum_{r=1}^{N_{\mathrm{R}}}\left|h_{r j, k}\right|^{2}}} .
$$

Meanwhile, the RX channel correlation $\rho_{\mathrm{RX}, i(k) j(l)}$ between the $i$-th RX antenna of the $k$-th user and $j$-th RX antenna of the $l$-th user is defined as follows:

Table A. 1 TX channel correlations when $\left(N_{\mathrm{T}} \times N_{\mathrm{R}} \times K\right)=(4 \times 2 \times 2)$, and $\Delta \theta=\pi / 10$. (Note that $\# i$ denotes the $i$-th TX antenna at the BS)

\begin{tabular}{|c|c|c|c|c|c||c|c|c|c|}
\hline \multicolumn{2}{|c|}{} & \multicolumn{4}{|c|}{ TX- $x / \mathrm{RX}-x$} & \multicolumn{4}{c|}{ TX- $y / \mathrm{RX}-y$} \\
\cline { 2 - 10 } \multicolumn{2}{c|}{} & $\# 1$ & $\# 2$ & $\# 3$ & $\# 4$ & $\# 1$ & $\# 2$ & $\# 3$ & $\# 4$ \\
\hline \hline \multirow{3}{*}{$@ \mathrm{BS}=0.5 \lambda$} & $\# 1$ & 1.00 & 0.97 & 0.88 & 0.75 & 1.00 & 0.58 & 0.26 & 0.70 \\
\cline { 2 - 10 } & $\# 2$ & 0.97 & 1.00 & 0.97 & 0.88 & 0.58 & 1.00 & 0.58 & 0.26 \\
\cline { 2 - 10 } & $\# 3$ & 0.88 & 0.97 & 1.00 & 0.97 & 0.26 & 0.58 & 1.00 & 0.58 \\
\cline { 2 - 10 } & $\# 4$ & 0.75 & 0.88 & 0.97 & 1.00 & 0.70 & 0.26 & 0.58 & 1.00 \\
\hline \hline \multirow{3}{*}{$@ \mathrm{BS}=1.0 \lambda$} & $\# 1$ & 1.00 & 0.88 & 0.57 & 0.18 & 1.00 & 0.26 & 0.48 & 0.12 \\
\cline { 2 - 10 } & $\# 2$ & 0.88 & 1.00 & 0.88 & 0.57 & 0.26 & 1.00 & 0.26 & 0.48 \\
\cline { 2 - 10 } & $\# 3$ & 0.57 & 0.88 & 1.00 & 0.88 & 0.48 & 0.26 & 1.00 & 0.26 \\
\cline { 2 - 10 } & $\# 4$ & 0.18 & 0.57 & 0.88 & 1.00 & 0.12 & 0.48 & 0.26 & 1.00 \\
\hline
\end{tabular}

Table A. 2 TX channel correlations when $\left(N_{\mathrm{T}} \times N_{\mathrm{R}} \times K\right)=(4 \times 2 \times 2)$, and $\Delta \theta=\pi / 4$. (Note that \#i denotes the $i$-th TX antenna at the BS)

\begin{tabular}{|c|c|c|c|c|c|c|c|c|c|}
\hline & \multicolumn{4}{|c|}{ TX- $x /$ RX- $x$} & \multicolumn{4}{|c|}{ TX-y/RX-y } \\
\hline & & $\# 1$ & $\# 2$ & $\# 3$ & $\# 4$ & $\# 1$ & $\# 2$ & $\# 3$ & $\# 4$ \\
\hline \multirow{4}{*}{$\begin{array}{l}@ \mathrm{BS}=0.5 \lambda \\
@ \mathrm{Us}=0.5 \lambda\end{array}$} & $\overline{\# 1}$ & 1.00 & 0.83 & 0.40 & 0.14 & 1.00 & 0.45 & 0.12 & 0.09 \\
\hline & $\# 2$ & 0.83 & 1.00 & 0.83 & 0.40 & 0.45 & 1.00 & 0.45 & 0.12 \\
\hline & $\# 3$ & 0.40 & 0.83 & 1.00 & 0.83 & 0.12 & 0.45 & 1.00 & 0.45 \\
\hline & \#4 & 0.14 & 0.40 & 0.83 & 1.00 & 0.09 & 0.12 & 0.45 & 1.00 \\
\hline \multirow{4}{*}{$\begin{array}{l}@ \mathrm{BS}=1.0 \lambda \\
@ \mathrm{Us}=0.5 \lambda\end{array}$} & \#1 & 1.00 & 0.40 & 0.39 & 0.16 & 21.00 & 0.12 & 0.11 & 0.16 \\
\hline & $\# 2$ & 0.40 & 1.00 & 0.40 & 0.39 & 0.12 & 1.00 & 0.12 & 0.11 \\
\hline & $\# 3$ & 0.39 & 0.40 & 1.00 & 0.40 & 0.11 & 0.12 & 1.00 & 0.12 \\
\hline & \#4 & 0.16 & 0.39 & 0.40 & 1.00 & 0.16 & 0.11 & 0.12 & 1.00 \\
\hline
\end{tabular}

Table A. 3 TX channel correlations when $\left(N_{\mathrm{T}} \times N_{\mathrm{R}} \times K\right)=(6 \times 3 \times 2)$, and $\Delta \theta=\pi / 10$. (Note that $\# i$ denotes the $i$-th TX antenna at the BS)

\begin{tabular}{|c|c|c|c|c|c|c|c|c|c|c|c|c|c|}
\hline & \multicolumn{6}{|c|}{ TX- $x /$ RX- $x$} & \multicolumn{6}{|c|}{ TX-y/RX-y } \\
\hline & & $\# 1$ & $\# 2$ & $\# 3$ & $\# 4$ & $\# 5$ & $\# 6$ & $\# 1$ & $\# 2$ & $\# 3$ & $\# 4$ & $\# 5$ & $\# 6$ \\
\hline \multirow{6}{*}{$\begin{array}{l}@ \mathrm{BS}=0.5 \lambda \\
@ \mathrm{Us}=0.5 \lambda\end{array}$} & 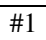 & $\overline{c 1.00}$ & 0.97 & $\overline{0.88}$ & $\overline{0.75}$ & $\overline{0.57}$ & 0.38 & 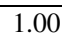 & $\overline{0.58}$ & $\overline{0.26}$ & $\overline{0.70}$ & $\overline{0.48}$ & $\overline{0.04}$ \\
\hline & $\# 2$ & 0.97 & 1.00 & 0.97 & 0.88 & 0.75 & 0.57 & 0.58 & 1.00 & 0.58 & 0.26 & 0.70 & 0.48 \\
\hline & $\# 3$ & 0.88 & 0.97 & 1.00 & 0.97 & 0.88 & 0.75 & 0.26 & 0.58 & 1.00 & 0.58 & 0.26 & 0.70 \\
\hline & $\# 4$ & 0.75 & 0.88 & 0.97 & 1.00 & 0.97 & 0.88 & 0.70 & 0.26 & 0.58 & 1.00 & 0.58 & 0.26 \\
\hline & $\# 5$ & 0.57 & 0.75 & 0.88 & 0.97 & 1.00 & 0.97 & 0.48 & 0.70 & 0.26 & 0.58 & 1.00 & 0.58 \\
\hline & $\# 6$ & 0.38 & 0.57 & 0.75 & 0.88 & 0.97 & 1.00 & 0.04 & 0.48 & 0.70 & 0.26 & 0.58 & 1.00 \\
\hline \multirow{6}{*}{$\begin{array}{l}@ \mathrm{BS}=1.0 \lambda \\
@ \mathrm{Us}=0.5 \lambda\end{array}$} & $\overline{7 \# 1}$ & 1.00 & 0.88 & 0.57 & 0.18 & 0.18 & 0.38 & 7.00 & 0.26 & 0.48 & 0.12 & 0.02 & 0.38 \\
\hline & $\# 2$ & 0.88 & 1.00 & 0.88 & 0.57 & 0.18 & 0.18 & 0.26 & 1.00 & 0.26 & 0.48 & 0.12 & 0.02 \\
\hline & $\# 3$ & 0.57 & 0.88 & 1.00 & 0.88 & 0.57 & 0.18 & 0.48 & 0.26 & 1.00 & 0.26 & 0.48 & 0.12 \\
\hline & $\# 4$ & 0.18 & 0.57 & 0.88 & 1.00 & 0.88 & 0.57 & 0.12 & 0.48 & 0.26 & 1.00 & 0.26 & 0.48 \\
\hline & $\# 5$ & 0.18 & 0.18 & 0.57 & 0.88 & 1.00 & 0.88 & 0.02 & 0.12 & 0.48 & 0.26 & 1.00 & 0.26 \\
\hline & \#6 & 0.38 & 0.18 & 0.18 & 0.57 & 0.88 & 1.00 & 0.38 & 0.02 & 0.12 & 0.48 & 0.26 & 1.00 \\
\hline
\end{tabular}


Table A. 4 TX channel correlations when $\left(N_{\mathrm{T}} \times N_{\mathrm{R}} \times K\right)=(6 \times 3 \times 2)$, and $\Delta \theta=\pi / 4$. (Note that $\# i$ denotes the $i$-th TX antenna at the BS)

\begin{tabular}{|c|c|c|c|c|c|c|c|c|c|c|c|c|c|}
\hline & \multicolumn{6}{|c|}{ TX $-x /$ RX $-x$} & \multicolumn{6}{|c|}{ TX-y/RX-y } \\
\hline & & $\# 1$ & $\# 2$ & $\# 3$ & $\# 4$ & $\# 5$ & $\# 6$ & $\# 1$ & $\# 2$ & $\# 3$ & $\# 4$ & $\# 5$ & \#6 \\
\hline \multirow{6}{*}{$\begin{array}{l}@ \mathrm{BS}=0.5 \lambda \\
@ \mathrm{Us}=0.5 \lambda\end{array}$} & \#1 & 1.00 & 0.83 & 0.40 & 0.14 & 0.39 & 0.38 & 1.00 & 0.45 & 0.12 & 0.09 & 0.11 & 0.23 \\
\hline & $\# 2$ & 0.83 & 1.00 & 0.83 & 0.40 & 0.14 & 0.39 & 0.45 & 1.00 & 0.45 & 0.12 & 0.09 & 0.11 \\
\hline & $\# 3$ & 0.40 & 0.83 & 1.00 & 0.83 & 0.40 & 0.14 & 0.12 & 0.45 & 1.00 & 0.45 & 0.12 & 0.09 \\
\hline & $\# 4$ & 0.14 & 0.40 & 0.83 & 1.00 & 0.83 & 0.40 & 0.09 & 0.12 & 0.45 & 1.00 & 0.45 & 0.12 \\
\hline & $\# 5$ & 0.39 & 0.14 & 0.40 & 0.83 & 1.00 & 0.83 & 0.11 & 0.09 & 0.12 & 0.45 & 1.00 & 0.45 \\
\hline & $\# 6$ & 0.38 & 0.39 & 0.14 & 0.40 & 0.83 & 1.00 & 0.23 & 0.11 & 0.09 & 0.12 & 0.45 & 1.00 \\
\hline \multirow{6}{*}{$\begin{array}{l}@ \mathrm{BS}=1.0 \lambda \\
@ \mathrm{Us}=0.5 \lambda\end{array}$} & $\# 1$ & 1.00 & 0.40 & 0.39 & 0.16 & 0.33 & 0.11 & 1.00 & 0.12 & 0.11 & $\overline{0.16}$ & 0.23 & $\overline{0.14}$ \\
\hline & $\# 2$ & 0.40 & 1.00 & 0.40 & 0.39 & 0.16 & 0.33 & 0.12 & 1.00 & 0.12 & 0.11 & 0.16 & 0.23 \\
\hline & $\# 3$ & 0.39 & 0.40 & 1.00 & 0.40 & 0.39 & 0.16 & 0.11 & 0.12 & 1.00 & 0.12 & 0.11 & 0.16 \\
\hline & $\# 4$ & 0.16 & 0.39 & 0.40 & 1.00 & 0.40 & 0.39 & 0.16 & 0.11 & 0.12 & 1.00 & 0.12 & 0.11 \\
\hline & $\# 5$ & 0.33 & 0.16 & 0.39 & 0.40 & 1.00 & 0.40 & 0.23 & 0.16 & 0.11 & 0.12 & 1.00 & 0.12 \\
\hline & $\# 6$ & 0.11 & 0.33 & 0.16 & 0.39 & 0.40 & 1.00 & 0.14 & 0.23 & 0.16 & 0.11 & 0.12 & 1.00 \\
\hline
\end{tabular}

Table A. 5 RX channel correlations when $\left(N_{\mathrm{T}} \times N_{\mathrm{R}} \times K\right)=(4 \times 2 \times 2)$, and $\Delta \theta=\pi / 10$. (Note that $\# i \mathrm{U} j$ denotes the $i$-th $\mathrm{RX}$ antenna at the $j$-th user)

\begin{tabular}{|c|c|c|c|c|c|c|c|c|c|}
\hline & \multicolumn{4}{|c|}{ TX- $x / \mathrm{RX}-x$} & \multicolumn{4}{|c|}{ TX-y/RX-y } \\
\hline & & \#1U1 & $\# 2 \mathrm{U} 1$ & $\# 1 \mathrm{U} 2$ & $\# 2 \mathrm{U} 2$ & \#1U1 & \#2U1 & \#1U2 & $\# 2 \mathrm{U} 2$ \\
\hline \multirow{4}{*}{$\begin{array}{l}@ \mathrm{BS}=0.5 \lambda \\
@ \mathrm{Us}=0.5 \lambda\end{array}$} & \#1U1 & 21.00 & 0.30 & 0.32 & 0.40 & 21.00 & 0.30 & 0.04 & 0.04 \\
\hline & $\# 2 \mathrm{U} 1$ & 0.30 & 1.00 & 0.14 & 0.32 & 0.30 & 1.00 & 0.07 & 0.04 \\
\hline & \#1U2 & 0.32 & 0.14 & 1.00 & 0.30 & 0.04 & 0.07 & 1.00 & 0.30 \\
\hline & \#2U2 & 0.40 & 0.32 & 0.30 & 1.00 & 0.04 & 0.04 & 0.30 & 1.00 \\
\hline \multirow{4}{*}{$\begin{array}{l}@ \mathrm{BS}=1.0 \lambda \\
@ \mathrm{Us}=0.5 \lambda\end{array}$} & \#1U1 & 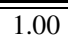 & 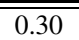 & 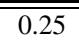 & $\overline{c 0.32}$ & 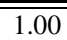 & 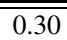 & 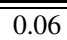 & 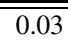 \\
\hline & \#2U1 & 0.30 & 1.00 & 0.14 & 0.25 & 0.30 & 1.00 & 0.06 & 0.06 \\
\hline & \#1U2 & 0.25 & 0.14 & 1.00 & 0.30 & 0.06 & 0.06 & 1.00 & 0.30 \\
\hline & \#2U2 & 0.32 & 0.25 & 0.30 & 1.00 & 0.03 & 0.06 & 0.30 & 1.00 \\
\hline
\end{tabular}

Table A. 6 RX channel correlations when $\left(N_{\mathrm{T}} \times N_{\mathrm{R}} \times K\right)=(4 \times 2 \times 2)$, and $\Delta \theta=\pi / 4$. (Note that $\# i \mathrm{U} j$ denotes the $i$-th $\mathrm{RX}$ antenna at the $j$-th user)

\begin{tabular}{|c|c|c|c|c|c|c|c|c|c|}
\hline & \multicolumn{4}{|c|}{ TX- $x / \mathrm{RX}-x$} & \multicolumn{4}{|c|}{ TX- $y / \mathrm{RX}-y$} \\
\hline & & \#1U1 & \#2U1 & \#1U2 & $\# 2 \mathrm{U} 2$ & \#1U1 & \#2U1 & \#1U2 & \#2U2 \\
\hline \multirow{4}{*}{$\begin{array}{l}@ \mathrm{BS}=0.5 \lambda \\
@ \mathrm{Us}=0.5 \lambda\end{array}$} & \#1U1 & 1.00 & 0.30 & 0.22 & 0.28 & $\overline{c 1.00}$ & 0.30 & 0.09 & 0.03 \\
\hline & $\# 2 \mathrm{U} 1$ & 0.30 & 1.00 & 0.14 & 0.22 & 0.30 & 1.00 & 0.02 & 0.09 \\
\hline & \#1U2 & 0.22 & 0.14 & 1.00 & 0.30 & 0.09 & 0.02 & 1.00 & 0.30 \\
\hline & $\# 2 \mathrm{U} 2$ & 0.28 & 0.22 & 0.30 & 1.00 & 0.03 & 0.09 & 0.30 & 1.00 \\
\hline \multirow{4}{*}{$\begin{array}{l}@ \mathrm{BS}=1.0 \lambda \\
@ \mathrm{Us}=0.5 \lambda\end{array}$} & \#1U1 & 1.00 & "0.30 & 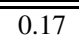 & 0.14 & 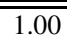 & 0.30 & 0.08 & 0.24 \\
\hline & $\# 2 \mathrm{U} 1$ & 0.30 & 1.00 & 0.14 & 0.17 & 0.30 & 1.00 & 0.10 & 0.08 \\
\hline & \#1U2 & 0.17 & 0.14 & 1.00 & 0.30 & 0.08 & 0.10 & 1.00 & 0.30 \\
\hline & \#2U2 & 0.14 & 0.17 & 0.30 & 1.00 & 0.24 & $\begin{array}{l}0.08 \\
\end{array}$ & 0.30 & 1.00 \\
\hline
\end{tabular}

$$
\rho_{\mathrm{RX}, i(k) j(l)}=\frac{\sum_{t=1}^{N_{\mathrm{T}}} h_{i t, k}^{*} h_{j t, l}}{\sqrt{\sum_{t=1}^{N_{\mathrm{T}}}\left|h_{i t, k}\right|^{2}} \sqrt{\sum_{t=1}^{N_{\mathrm{T}}}\left|h_{j t, l}\right|^{2}}}
$$

where $1 \leq k, l \leq K$. Note that in the above equation, when $k=l$ we have the $\mathrm{RX}$ intra-user channel correlations, i.e., correlations between RX antennas for the same user, and when $k \neq l$ we have the $\mathrm{RX}$ inter-user channel correlations, i.e., correlations between RX antennas of two different users. It should also be noted that the absolute values of $\rho_{\mathrm{TX}, i j}$ and $\rho_{\mathrm{RX}, i(k) j(l)}$ are in the range from 0 to 1 , $\left|\rho_{\mathrm{TX}, i j}\right|=\left|\rho_{\mathrm{TX}, j i}\right|$, and $\left|\rho_{\mathrm{RX}, i(k) j(l)}\right|=\left|\rho_{\mathrm{RX}, j(l) i(k)}\right|$.

The TX channel correlations between TX antennas at the BS when $\left(N_{\mathrm{T}} \times N_{\mathrm{R}} \times K\right)=(4 \times 2 \times 2) \&(6 \times 3 \times 2)$ and $\Delta \theta=\pi / 10 \& \pi / 4$ are shown in Tables A. $1, \mathrm{~A} \cdot 2, \mathrm{~A} \cdot 3$, and A.4. The correlations are higher for the TX-x/RX-x orientation than for the TX-y/RX-y orientation. This can be proved as follows: The configuration of the RX array does not affect the TX channel correlations. The point is the configuration of TX antennas at the BS. Also, it should be noted that User 1 and User 2 are located symmetrically about the $x$-axis as shown in Fig. 5 . Let us consider channels of $h_{r i, 1}, h_{r j, 1}, h_{r i, 2}$, and $h_{r j, 2}$. Statistically, we have samples that satisfy $h_{r i, 2}=h_{r i, 1}$, and $h_{r j, 2}=h_{r j, 1}$ for the TX- $x$ configuration. Similarly, we have samples that satisfy $h_{r i, 2}=h_{r i, 1}^{*}$, and $h_{r j, 2}=h_{r j, 1}^{*}$ for the TX-y configuration. For the above samples of channel values, we have the following equations: 
Table A. 7 RX channel correlations when $\left(N_{\mathrm{T}} \times N_{\mathrm{R}} \times K\right)=(6 \times 3 \times 2)$, and $\Delta \theta=\pi / 10$. (Note that $\# i \mathrm{U} j$ denotes the $i$-th $\mathrm{RX}$ antenna at the $j$-th user)

\begin{tabular}{|c|c|c|c|c|c|c|c|c|c|c|c|c|c|}
\hline & \multicolumn{6}{|c|}{ TX- $x / \mathrm{RX}-x$} & \multicolumn{6}{|c|}{ TX-y/RX-y } \\
\hline & & $\# 1 \mathrm{U1}$ & $\# 2 \mathrm{U} 1$ & $\# 3 \mathrm{U} 1$ & \#1U2 & $\# 2 \mathrm{U} 2$ & $\# 3 \mathrm{U} 2$ & $\# 1 \mathrm{U} 1$ & $\# 2 \mathrm{U} 1$ & $\# 3 \mathrm{U} 1$ & \#1U2 & $\# 2 \mathrm{U} 2$ & $\# 3 \mathrm{U} 2$ \\
\hline \multirow{6}{*}{$\begin{array}{l}@ \mathrm{BS}=0.5 \lambda \\
@ \mathrm{Us}=0.5 \lambda\end{array}$} & \#1U1 & 1.00 & 0.30 & 0.22 & 0.29 & 0.36 & 0.26 & 1.00 & 0.30 & 0.22 & 0.05 & 0.04 & 0.06 \\
\hline & $\# 2 \mathrm{U} 1$ & 0.30 & 1.00 & 0.30 & 0.14 & 0.29 & 0.36 & 0.30 & 1.00 & 0.30 & 0.06 & 0.05 & 0.04 \\
\hline & $\# 3 \mathrm{U} 1$ & 0.22 & 0.30 & 1.00 & 0.13 & 0.14 & 0.29 & 0.22 & 0.30 & 1.00 & 0.03 & 0.06 & 0.05 \\
\hline & \#1U2 & 0.29 & 0.14 & 0.13 & 1.00 & 0.30 & 0.22 & 0.05 & 0.06 & 0.03 & 1.00 & 0.30 & 0.22 \\
\hline & \#2U2 & 0.36 & 0.29 & 0.14 & 0.30 & 1.00 & 0.30 & 0.04 & 0.05 & 0.06 & 0.30 & 1.00 & 0.30 \\
\hline & \#3U2 & 0.26 & 0.36 & 0.29 & 0.22 & 0.30 & 1.00 & 0.06 & 0.04 & 0.05 & 0.22 & 0.30 & 1.00 \\
\hline \multirow{6}{*}{$\begin{array}{l}@ \mathrm{BS}=1.0 \lambda \\
@ \mathrm{Us}=0.5 \lambda\end{array}$} & 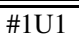 & 1.00 & 0.30 & 0.22 & 0.19 & 0.23 & $\overline{0.22}$ & $\overline{1.00}$ & 0.30 & 0.22 & 0.05 & 0.08 & $\overline{0.05}$ \\
\hline & $\# 2 \mathrm{U} 1$ & 0.30 & 1.00 & 0.30 & 0.14 & 0.19 & 0.23 & 0.30 & 1.00 & 0.30 & 0.02 & 0.05 & 0.08 \\
\hline & \#3U1 & 0.22 & 0.30 & 1.00 & 0.09 & 0.14 & 0.19 & 0.22 & 0.30 & 1.00 & 0.03 & 0.02 & 0.05 \\
\hline & \#1U2 & 0.19 & 0.14 & 0.09 & 1.00 & 0.30 & 0.22 & 0.05 & 0.02 & 0.03 & 1.00 & 0.30 & 0.22 \\
\hline & $\# 2 \mathrm{U} 2$ & 0.23 & 0.19 & 0.14 & 0.30 & 1.00 & 0.30 & 0.08 & 0.05 & 0.02 & 0.30 & 1.00 & 0.30 \\
\hline & $\# 3 \mathrm{U} 2$ & 0.22 & 0.23 & 0.19 & 0.22 & 0.30 & 1.00 & 0.05 & 0.08 & 0.05 & 0.22 & 0.30 & 1.00 \\
\hline
\end{tabular}

Table A. 8 RX channel correlations when $\left(N_{\mathrm{T}} \times N_{\mathrm{R}} \times K\right)=(6 \times 3 \times 2)$, and $\Delta \theta=\pi / 4$. (Note that $\# i \mathrm{U} j$ denotes the $i$-th $\mathrm{RX}$ antenna at the $j$-th user)

\begin{tabular}{|c|c|c|c|c|c|c|c|c|c|c|c|c|c|}
\hline & \multicolumn{6}{|c|}{$\mathrm{TX}-x / \mathrm{RX}-x$} & \multicolumn{6}{|c|}{ TX-y/RX- $y$} \\
\hline & & \#1U1 & \#2U1 & \#3U1 & \#1U2 & \#2U2 & \#3U2 & \#1U1 & $\# 2 \mathrm{U} 1$ & \#3U1 & \#1U2 & $\# 2 \mathrm{U} 2$ & \#3U2 \\
\hline \multirow{6}{*}{$\begin{array}{l}@ \mathrm{BS}=0.5 \lambda \\
@ \mathrm{Us}=0.5 \lambda\end{array}$} & \#1U1 & 1.00 & 0.30 & 0.22 & 0.17 & 0.18 & 0.22 & $\overline{1.00}$ & $\overline{0.30}$ & 0.22 & 0.03 & 0.02 & 0.05 \\
\hline & $\# 2 \mathrm{U} 1$ & 0.30 & 1.00 & 0.30 & 0.14 & 0.17 & 0.18 & 0.30 & 1.00 & 0.30 & 0.11 & 0.03 & 0.02 \\
\hline & \#3U1 & 0.22 & 0.30 & 1.00 & 0.09 & 0.14 & 0.17 & 0.22 & 0.30 & 1.00 & 0.04 & 0.11 & 0.03 \\
\hline & \#1U2 & 0.17 & 0.14 & 0.09 & 1.00 & 0.30 & 0.22 & 0.03 & 0.11 & 0.04 & 1.00 & 0.30 & 0.22 \\
\hline & \#2U2 & 0.18 & 0.17 & 0.14 & 0.30 & 1.00 & 0.30 & 0.02 & 0.03 & 0.11 & 0.30 & 1.00 & 0.30 \\
\hline & $\# 3 \mathrm{U} 2$ & 0.22 & 0.18 & 0.17 & 0.22 & 0.30 & 1.00 & 0.05 & 0.02 & 0.03 & 0.22 & 0.30 & 1.00 \\
\hline \multirow{6}{*}{$\begin{array}{l}@ \mathrm{BS}=1.0 \lambda \\
@ \mathrm{Us}=0.5 \lambda\end{array}$} & \#1U1 & 1.00 & 0.30 & 0.22 & 0.12 & 0.13 & 0.11 & 1.00 & 0.30 & 0.22 & 0.05 & 0.15 & 0.02 \\
\hline & \#2U1 & 0.30 & 1.00 & 0.30 & 0.13 & 0.12 & 0.13 & 0.30 & 1.00 & 0.30 & 0.08 & 0.05 & 0.15 \\
\hline & \#3U1 & 0.22 & 0.30 & 1.00 & 0.15 & 0.13 & 0.12 & 0.22 & 0.30 & 1.00 & 0.03 & 0.08 & 0.05 \\
\hline & \#1U2 & 0.12 & 0.13 & 0.15 & 1.00 & 0.30 & 0.22 & 0.05 & 0.08 & 0.03 & 1.00 & 0.30 & 0.22 \\
\hline & \#2U2 & 0.13 & 0.12 & 0.13 & 0.30 & 1.00 & 0.30 & 0.15 & 0.05 & 0.08 & 0.30 & 1.00 & 0.30 \\
\hline & \#3U2 & 0.11 & 0.13 & 0.12 & 0.22 & 0.30 & 1.00 & 0.02 & 0.15 & 0.05 & 0.22 & 0.30 & 1.00 \\
\hline
\end{tabular}

$$
\begin{aligned}
& X_{1}(r)=\sum_{k=1}^{2} h_{r i, k}^{*} h_{r j, k}=2 h_{r i, 1}^{*} h_{r j, 1} \\
& \text { for the TX-x case } \\
& Y_{1}(r)=\sum_{k=1}^{2} h_{r i, k}^{*} h_{r j, k}=h_{r i, 1}^{*} h_{r j, 1}+h_{r i, 1} h_{r j, 1}^{*}
\end{aligned}
$$

\section{for the TX-y case}

It is easily seen that $Y_{1}(r)$ is a real-valued number, and that the real part of $X_{1}(r)$ is $Y_{1}(r)$. Then, we have $\left|\sum_{r=1}^{N_{\mathrm{R}}} Y_{1}(r)\right| \leq$ $\left|\sum_{r=1}^{N_{\mathrm{R}}} X_{1}(r)\right|$. From this inequality and the Eq. (A. 1), we can see that $\rho_{\mathrm{TX}, i j}$ has a higher value for the $\mathrm{TX}-x / \mathrm{RX}-x$ case than for the TX- $y / \mathrm{RX}-y$ case. It is also observed that when $\mathrm{AS}$ at the BS is extended from $0.5 \lambda$ to $1.0 \lambda$, the correlations for both the array orientations significantly decrease. In addition, when angle spread $\Delta \theta$ from the BS to each ring of scatterers is increased from $\pi / 10$ to $\pi / 4$, the correlations considerably reduce.

Different from the single-user MIMO case, for the multiuser MIMO systems, both intra-user and inter-user channel correlations should be considered. The RX intra-user and inter-user channel correlations when $\left(N_{\mathrm{T}} \times N_{\mathrm{R}} \times K\right)=$ $(4 \times 2 \times 2) \&(6 \times 3 \times 2)$ and $\Delta \theta=\pi / 10 \& \pi / 4$ are shown in Tables A. 5, A. 6, A. 7, and A. 8. The RX correlations are much smaller than the TX ones. This is because scatterers are uniformly distributed around each user, hence the angle spread from the RX arrays to rings of scatterers is equal to $2 \pi$. The larger the angle spread, the smaller the correlation. It is interesting that the RX intra-user correlations for two users are the same and do not depend on the changes of the array orientation at both the BS and users, the antenna spacing at the BS, and the angle spread from the BS to the rings of scatterers. The reason for this is because the angle spread for the RX is $2 \pi$ independently of the RX array orientation. It should be noted that the environment concerned here is Rayleigh fading, i.e., there are not line-of-sight waves from the BS to users. While the intra-user correlations are constant, the inter-user ones are dependent on the array orientation and the changes at the BS. Lower inter-user correlations can be seen for the TX- $y / \mathrm{RX}-y$ orientation compared with the TX- $x / \operatorname{RX}-x$ orientation. In addition, when AS at the BS is extended or when the TX angle spread increases, inter-user correlations reduce more for the TX- $x / \mathrm{RX}-x$ orientation than for the TX- $y / \mathrm{RX}-y$ orientation. 


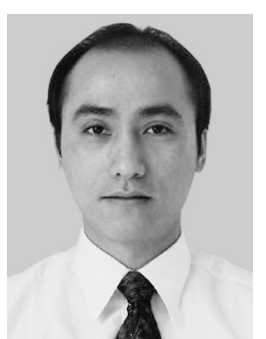

Huu Phu Bui received B.S., M.S., and $\mathrm{Ph} . \mathrm{D}$. degrees in electronics engineering from Danang University, Hochiminh University, Vietnam, and Hokkaido University, Japan in 1997, 2002, and 2007, respectively. From 1997 to 2007, he was with Radio Frequency Directorate, Ministry of Information and Communications, Vietnam. From 2007, he has been with University of Natural Sciences, Vietnam. He is currently a postdoctoral reseacher in Hokkaido University, Japan. His research interests are in channel prediction and signal processing for MIMO systems. He received IEEE VTS Japan Chapter Young Researcher's Encouragement Award in 2006. Dr. Bui is a member of the IEEE.

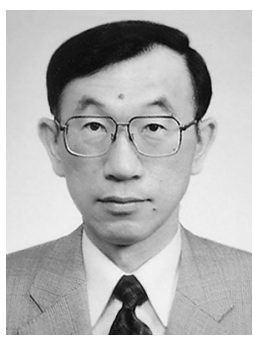

Yasutaka Ogawa received the B.E., M.E. and Ph.D. degrees from Hokkaido University, Sapporo, Japan, in 1973, 1975, and 1978, respectively. Since 1979, he has been with Hokkaido University, where he is currently a Professor of the Graduate School of Information Science and Technology. During 1992-1993, he was with ElectroScience Laboratory, the Ohio State University, U.S.A., as a Visiting Scholar, on leave from Hokkaido University. His interests are in adaptive antennas, mobile communications, super-resolution techniques, and MIMO systems. Dr. Ogawa received the Yasujiro Niwa outstanding paper award in 1978, the Young Researchers' Award of IEICE Japan in 1982, and the Best Paper Award from IEICE Japan in 2007. Dr. Ogawa is a senior member of the IEEE.

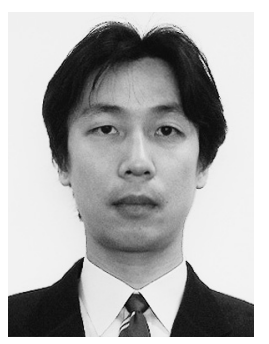

Toshihiko Nishimura received the B.S. and M.S. degrees in physics and Ph.D. degree in electronics engineering from Hokkaido University, Sapporo, Japan, in 1992, 1994, and 1997, respectively. In 1998, he joined the Graduate School of Engineering (reorganized to Graduate School of Information Science and Technology at present) at Hokkaido University, where he is currently an Assistant Professor of the Graduate School of Information Science and Technology. His current research interests are in MIMO systems using smart antenna techniques. Dr. Nishimura received the Young Researchers' Award of IEICE Japan in 2000, and the Best Paper Award from IEICE Japan in 2007. Dr. Nishimura is a member of the IEEE.

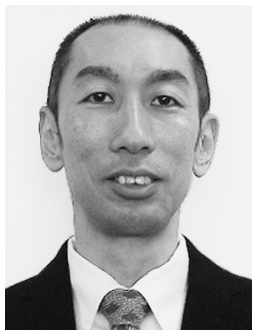

Takeo Ohgane received the B.E., M.E., and $\mathrm{Ph} . \mathrm{D}$. degrees in electronics engineering from Hokkaido University, Sapporo, Japan, in 1984, 1986, and 1994, respectively. From 1986 to 1992, he was with Communications Research Laboratory, Ministry of Posts and Telecommunications. From 1992 to 1995, he was on assignment at ATR Optical and Radio Communications Research Laboratory. Since 1995, he has been with Hokkaido University, where he is an Associate Professor. During 2005-2006, he was at Centre for Communications Research, University of Bristol, U.K., as a Visiting Fellow. His interests are in MIMO signal processing for wireless communications. Dr. Ohgane received the IEEE AP-S Tokyo Chapter Young Engineer Award in 1993, the Young Researchers' Award of IEICE Japan in 1990, and the Best Paper Award from IEICE Japan in 2007. Dr. Ohgane is a member of the IEEE. 